\title{
Rapamycin Attenuated Cardiac Hypertrophy Induced by Isoproterenol and Maintained Energy Homeostasis via Inhibiting NF- $\kappa$ B Activation
}

\author{
Xi Chen, ${ }^{1}$ Siyu Zeng, ${ }^{1}$ Jian Zou, ${ }^{2}$ Yanfang Chen, ${ }^{3}$ Zhongbao Yue, ${ }^{1}$ \\ Ying Gao, ${ }^{1}$ Luankun Zhang, ${ }^{1}$ Weiwei Cao, ${ }^{1}$ and Peiqing Liu ${ }^{1}$ \\ ${ }^{1}$ Department of Pharmacology and Toxicology, School of Pharmaceutical Sciences, Sun Yat-sen University, Guangzhou 510006, China \\ ${ }^{2}$ Department of Pharmacy, Chengdu Fifth People's Hospital, Chengdu 611130, China \\ ${ }^{3}$ The Second Affiliated Hospital of Guangzhou Medical University, Guangzhou 510260, China
}

Correspondence should be addressed to Peiqing Liu; liupq@mail.sysu.edu.cn

Received 10 March 2014; Revised 11 May 2014; Accepted 14 May 2014; Published 19 June 2014

Academic Editor: Elaine Hatanaka

Copyright (c) 2014 Xi Chen et al. This is an open access article distributed under the Creative Commons Attribution License, which permits unrestricted use, distribution, and reproduction in any medium, provided the original work is properly cited.

\begin{abstract}
Rapamycin, also known as sirolimus, is an immunosuppressant drug used to prevent rejection organ (especially kidney) transplantation. However, little is known about the role of Rapa in cardiac hypertrophy induced by isoproterenol and its underlying mechanism. In this study, Rapa was administrated intraperitoneally for one week after the rat model of cardiac hypertrophy induced by isoproterenol established. Rapa was demonstrated to attenuate isoproterenol-induced cardiac hypertrophy, maintain the structure integrity and functional performance of mitochondria, and upregulate genes related to fatty acid metabolism in hypertrophied hearts. To further study the implication of NF- $\kappa \mathrm{B}$ in the protective role of Rapa, cardiomyocytes were pretreated with TNF- $\alpha$ or transfected with siRNA against NF- $\kappa \mathrm{B} / \mathrm{p} 65$ subunit. It was revealed that the upregulation of extracellular circulating proinflammatory cytokines induced by isoproterenol was able to be reversed by Rapa, which was dependent on NF- $\kappa \mathrm{B}$ pathway. Furthermore, the regression of cardiac hypertrophy and maintaining energy homeostasis by Rapa in cardiomyocytes may be attributed to the inactivation of NF- $\kappa \mathrm{B}$. Our results shed new light on mechanisms underlying the protective role of Rapa against cardiac hypertrophy induced by isoproterenol, suggesting that blocking proinflammatory response by Rapa might contribute to the maintenance of energy homeostasis during the progression of cardiac hypertrophy.
\end{abstract}

\section{Introduction}

Cardiac hypertrophy was induced by many kinds of physiological and pathophysiological stimuli, including exercise, pressure or volume overload, endocrine disorders, and valvular heart diseases [1]. Although it has been viewed as a temporary compensatory mechanism for hearts to diminish wall stress, cardiac hypertrophy is associated with increased risks of developing decompensatory heart failure [2].

Hearts consume more energy than any other organs to maintain excitation-contraction coupling. As a result, common heart diseases, either myocardial ischemia or myocardial infarction, were characterized by energy metabolism dysregulation. It has been demonstrated that fatty acid utilization was decreased significantly, resulting in energy insufficiency that exacerbated the progression of heart diseases [3]. The reduction in fatty oxidation led to the abnormal accumulation of intracellular lipid, which could be lipotoxic to hearts [4]. Mitochondria provide ATP to hearts for preserving contractile and relaxation functions through conversion of metabolism substrates to usable energy [5]. Thus, it is of great importance for hearts to maintain structure integrity and functional performance of mitochondria, as well as the efficiency in utilization of fatty acid [6].

Inducements of cardiac hypertrophy are of great complexities. The retention of salt and water, as well as excessive peripheral vasoconstriction, was to blame for the disease progression. And prior to overtly symptomatic decompensatory cardiac hypertrophy, sympathetic nervous system (SNS) activation was induced to increase the cardiac output at the onset 
of heart disease [7]; however, the persistent stimulation of neurohormonal factors contributes to the mechanical dysfunction of hearts ultimately [8]. It had been demonstrated that overexpression of biologically active neurohormonal molecules contributed to the progression of decompensated cardiac hypertrophy independently of the hemodynamic status, by virtue of the direct toxic effects of these neurohormonal molecules exert on hearts [9]. Isoproterenol is one of these biologically active neurohormonal molecules. In addition to its direct cardiac effect, isoproterenol was proved to have a cardiac trophic effect via stimulation of the renin angiotensin system (RAS) [10].

Rapamycin is a clinically used immunosuppressor in preventing transplant rejection [11] and restenosis in coronary arteries following balloon angioplasty. Recently, Rapa was demonstrated to ameliorate cardiac remodeling and improve the left ventricular function after myocardial infarction [12]. Besides, it has been reported that Rapa regressed left ventricular hypertrophy in renal transplant recipients regardless of blood pressure changes, suggesting nonhemodynamic-effect mechanisms of rapamycin on left ventricular mass [13]. Thus, it is worthy of investigation that whether the treatment of Rapa was capable of regressing cardiac hypertrophy induced by persistent stimulation of sympathetic nervous system. And the underlying mechanisms of Rapa in protecting against cardiac hypertrophy induced by isoproterenol remain to be elucidated.

Based on these considerations mentioned above, we established the rat model of cardiac hypertrophy induced by isoproterenol, and then Rapa was administrated to evaluate the cardiac function and mitochondrial functional performance. In this study, Rapa was observed to attenuate isoproterenol-induced cardiac hypertrophy and maintain energy homeostasis in adult rats. Increased levels of proinflammatory cytokines were detected in cardiomyocytes in response to isoproterenol, which could be substantially attenuated by Rapa. Moreover, these protective effects of Rapa might be attributed to the inactivation of NF- $\kappa$ B. The present data revealed that the role of Rapa in protecting hearts from isoproterenol-induced cardiac hypertrophy and maintaining energy homeostasis was dependent on NF- $\kappa$ B pathway.

\section{Materials and Methods}

2.1. Animal Models of Cardiac Hypertrophy and Experimental Protocols. Male Sprague-Dawley rats weighing $225 \mathrm{~g}$ to $250 \mathrm{~g}$ were purchased from Animal Breeding Center of Sun Yatsen University. Isoproterenol (5.5 mg/Kg, Merck) dissolved in saline was injected subcutaneously for consecutive ten days. Control animals underwent the same procedure, except that saline vehicle was injected subcutaneously instead of isoproterenol. After the infusion of isoproterenol, rats surviving were assigned to the following groups randomly: a vehicle-treated group (vehicle, $n=6$ ), a rapamycin-treated group $(1.2 \mathrm{mg} / \mathrm{kg}$, Selleck; $n=6)$. Treatments of Rapa were injected intraperitoneally daily for seven days. The doses we administrated here were comparable with those used in mice studies when normalized by body surface area $[12,14]$. The experiments were approved by the ethics Committees of Sun Yat-sen University of medical Science.

2.2. Echocardiographic Studies and Histological Analysis. Rats were anesthetized with $2 \%$ isoflurane allowing spontaneous breathing. The Vevo 2100 scanner equipped with a $16 \mathrm{MHz}$ Probe (Visual Sonics, Toronto, Canada) was used to perform echocardiography analysis. After a good-quality twodimensional image was obtained, M-mode images of left ventricles were recorded. Left ventricular anterior and posterior wall thickness (LVAW and LVPW), fractional shortening (LVFS), and ejection fraction (LVEF) were measured and analyzed.

Once all rats were sacrificed, the hearts were washed with PBS and dried to weigh and fixed within $4 \%$ paraform overnight. Paraffin-embedded sections $(7 \mu \mathrm{m})$ were stained with hematoxylin and eosin. Slides were examined with brightfield microscopy and $\times 400$ images were captured. The mean myocyte diameter was calculated by randomly measuring 100 cardiomyocytes in 10 randomly chosen fields according to method and criteria reported $[15,16]$.

2.3. Transmission Electron Microscopy Analysis. Cardiac tissues were cut into $1 \mathrm{~mm}$ cubes immediately after sacrifice and fixed in $2.5 \%$ glutaraldehyde and then postfixed in $1 \%$ osmium tetroxide. After acetone dehydration and embedding, the ultrathin sections were stained as methods described [17]. Images were taken at $120 \mathrm{KV}$ with JEM1400 electron microscopy equipped with a high-performance CCD camera.

2.4. Mitochondrial Oxygen Consumption. Hearts were freshly excised, and the apex of left ventricle $(200 \mathrm{mg})$ was obtained from hearts in three experimental groups. Then fresh mitochondria were isolated using mitochondria extraction and isolation kit (GenMed Scientifics) according to the manufacturer's instruction. Clark-type oxygen electrode (Strathkelvin, Scotland) was used to determine the rate of mitochondrial respiration $\left(25^{\circ} \mathrm{C}\right)$. The respiration medium was prepared as method reported [18]. Following the addition of $1 \mathrm{mM}$ adenosine diphosphate (ADP), the state III respiration was recorded. After the complete phosphorylation of the added ADP, the state IV respiration was recorded as well. The respiration control rate (RCR), which reflects the function of mitochondria, was represented by the ratio of rate of state III respiration to the rate of state IV respiration. Mitochondrial protein concentration was determined by BCA protein assay kit (Thermo Scientifics). The rates of oxygen consumption were expressed as nmol $\mathrm{O}_{2} / \mathrm{min} / \mathrm{mg}$.

2.5. Quantitative Real-Time Polymerase Chain Reaction ( $q R T$ $P C R)$. According to the manufacturer's instruction, myocardial RNA was extracted from snap-frozen tissues with Trizol reagent (Invitrogen). The SYBR-Green Quantitative PCR kit (Takara) was used to determine the mRNA expression levels of each target gene by iCycler iQ system (Bio-Rad). All PCR reactions were performed in triplicate. Specific primer 
TABLE 1: Primer sequences for qRT-PCR.

\begin{tabular}{|c|c|}
\hline Target gene & Sequence \\
\hline \multirow{2}{*}{ ANF } & Forward: $5^{\prime}$-GGAAGTCAACCCGTCTCA-3' \\
\hline & Reverse: 5'-AGCCCTCAGTTTGCTTTT-3' \\
\hline \multirow{2}{*}{ BNP } & Forward: 5'-TTTGGGCAGAAGATAGACCG-3' \\
\hline & Reverse: 5'-AGAAGAGCCGCAGGCAGAG-3' \\
\hline \multirow{2}{*}{$\beta$-MHC } & Forward: 5'-GACAACGCCTATCAGTACATG-3' \\
\hline & Reverse: $5^{\prime}$-TGGCAGCAATAACAGCAAAA-3' \\
\hline \multirow{2}{*}{ GAPDH } & Forward: $5^{\prime}$-AGGAGTAAGAAACCCTGGAC-3' \\
\hline & Reverse: $5^{\prime}$-CTGGGATGGAATTGTGAG-3' \\
\hline \multirow{2}{*}{$\mathrm{CPT}-1 \beta$} & Forward: 5' -TCAAGGTTTGGCTCTATGAGGGCT-3' \\
\hline & Reverse: 5'-TCCAGGGACATCTTGTTCTTGCCA-3' \\
\hline \multirow{2}{*}{ СРТ-2 } & Forward: $5^{\prime}$-TCCTGCATACCAGCAGATGAACCA-3' \\
\hline & Reverse: 5'-TATGCAATGCCAAAGCCATCAGGG-3' \\
\hline \multirow{2}{*}{ MCAD } & Forward: $5^{\prime}$-CTGCTCGCAGAAATGGCGATGAAA-3' \\
\hline & Reverse: 5'-CAAAGGCCTTCGCAATAGAGGCAA-3’ \\
\hline \multirow{2}{*}{ LCAD } & Forward: $5^{\prime}$-AATGGGAGAAAGCCGGAGAAGTGA-3' \\
\hline & Reverse: 5'-GATGCCGCCATGTTTCTCTGCAAT-3' \\
\hline \multirow{2}{*}{ IL-1 $\beta$} & Forward: $5^{\prime}$-TCCTCTGTGACTCGTGGGAT-3' \\
\hline & Reverse: 5'-TCAGACAGCACGAGGCATTT-3' \\
\hline \multirow{2}{*}{ IL-2 } & Forward: $5^{\prime}$-CCAAGCAGGCCACAGAATTG-3' \\
\hline & Reverse: 5'-TCCAGCGTCTTCCAAGTGAA-3' \\
\hline \multirow{2}{*}{ TNF- $\alpha$} & Forward: 5' -TGGCGTGTTCATCCGTTCTC-3' \\
\hline & Reverse: 5'-CCCAGAGCCACAATTCCCTT-3' \\
\hline \multirow{2}{*}{ PGC-1 $\alpha$} & Forward: $5^{\prime}$-ACGAAAGGCTCAAGAGGGACGAAT-3' \\
\hline & Reverse: 5'-CACGGCGCTCTTCAATTGCTTTCT-3' \\
\hline \multirow{2}{*}{$\operatorname{PPAR} \alpha$} & Forward: 5'-AGCTCAGGACACAAGACGTTGTCA-3' \\
\hline & Reverse: 5'-AGGGACTTTCCAGGTCATCTGCTT-3' \\
\hline \multirow{2}{*}{$\beta$-actin } & Forward: $5^{\prime}$-TCGTGCGTGACATTAAAGAG-3' \\
\hline & Reverse: 5'-ATTGCCGATAGTGATGACCT-3' \\
\hline
\end{tabular}

sequences synthesized by Invitrogen were listed in Table 1. GAPDH and $\beta$-actin served as an endogenous control.

2.6. ATP Concentration Measurements. Using an ATP bioluminescent assay kit (Sigma), myocardial ATP concentration was measured according to the manufacturer's recommendations. Experiments were done in triplicate for each group.

2.7. Western Blot Analysis. Using a commercial available Nuclear and Cytoplasm Extraction Kit (Active Motif), nuclear proteins were isolated from left ventricles of rats or primary neonatal rat ventricular cardiomyocytes. The homogenate proteins were separated on SDS-PAGE and then transferred to PVDF membrane (Millipore). The membranes were incubated with primary polyclonal antibodies against p65 (Cell Signal Technology), I $\kappa$ B- $\alpha$ (Cell Signal Technology), Histone-H3 (Sigma), p-mTOR (Cell Signal Technology), mTOR (Cell Signal Technology), PPAR $\alpha$ (Sigma), PGC$1 \alpha$ (Calbiochem), GAPDH (Sigma), and $\alpha$-tubulin (Sigma) overnight at $4^{\circ} \mathrm{C}$. After washing in TBS-T buffer, the second antibodies (Promega) were conjugated at room temperature, and then protein bands were visualized using enhanced Super-Signal West Pico substrates (Pierce).

2.8. Primary Culture of Neonatal Rat Ventricular Cardiomyocytes and Treatments. Primary culture of neonatal rat ventricular cardiomyocytes was prepared using 1- to 3-day-old Sprague-Dawley rats as methods we reported [19]. Cells were cultured in $10 \%$ serum for 24 hours after isolation. 48 hours later, the serum-containing culture medium was replaced with DMEM containing $0.1 \%$ FBS. After incubation for 24 hours, the cells were further treated with isoproterenol (1 $\mu \mathrm{mol} / \mathrm{L})$, or in combination with rapamycin $(100 \mathrm{nmol} / \mathrm{L})$ was administrated to cells and maintained for 24 hours.

2.9. Small Interference RNA. The small interference RNA (siRNA) sequences against rat NF- $\kappa \mathrm{B}$ p65 subunit were synthesized by GenePharma. NF- $\kappa \mathrm{B}$ p 65 antisense sequences were $5^{\prime}$-UCUAUGGGAACUUGAAAGGTT-3'; the scrambled sequence was used as a negative control (NC). Small interference RNA (100 nmol/L) was transiently transfected into cardiomyocytes by adding it to lipofectamine 2000 
(Invitrogen) according to the manufacturer's instructions. The knockdown of p65 protein expression was confirmed by western blot analysis.

2.10. TNF- $\alpha, I L-1 \beta$, and $I L-2$ Measurement. An ELISA kit (Dakewe) was used to determine the concentrations of IL$1 \beta$, IL- 2 , and TNF- $\alpha$ in the culture medium according to the manufacturer's instructions.

2.11. Cell Surface Area Quantification. To determine the cell surface area, cells were fixed with $4 \%$ paraform and then permeabilized within $0.5 \%$ TritonX-100, after which cells were incubated with TRITC-labeled phalloidin (Sigma) for $30 \mathrm{~min}$ at room temperature as methods reported [20].

\subsection{Mitochondrial Membrane Potential Analysis. TMRE} (10 nmol/L), a mitochondrial potential-indicating fluorophore (Invitrogen, Molecular Probes), was added to cells for $30 \mathrm{~min}$, after which cells were visualized under confocal laser scanning microscopy (LSM710, Zeiss). Images were analyzed as reported previously [21].

2.13. Statistical Analysis. Data were expressed as mean $\pm \mathrm{SD}$. All results were obtained from three independent experiments. SPSS 17.0 for statistic software was used to perform all data analyses. Statistical significance of difference among experimental groups was assessed with one-way analysis of variance (ANOVA) followed by the Bonferroni post hoc test. The level of $P<0.05$ was considered to be statistically significant in all cases.

\section{Results}

3.1. Rapa Attenuated Cardiac Hypertrophy Induced by Isoproterenol in Adult Rats. We first determined whether the treatment of Rapa after consecutive isoproterenol-infusion for ten days reverses indices of myocardial hypertrophy. The ratios of heart weight to body weight $(\mathrm{HW} / \mathrm{BW})$ and left ventricular weight to body weight (LVW/BW) were significantly upregulated in isoproterenol-infusion model group compared with those in control group $(P<0.01$ versus control). As shown in Figure 1(a), rats treated with Rapa reduced the isoproterenolinduced increase in the ratios of both $\mathrm{HW} / \mathrm{BW}$ and LVW/BW significantly (HW/BW: $2.46 \pm 0.35$ versus $3.15 \pm 0.39 ; P<$ 0.01 versus model; $L V W / B W: 1.66 \pm 0.28$ versus $2.40 \pm 0.55$; $P<0.01$ versus model). Histological analysis revealed that hearts under isoproterenol stimulation demonstrated direct evidence of increased mean myocyte diameters, which was reversed in the Rapa-treated hearts (Figures 1(b) and 1(c)). Consistently, echocardiographic analysis showed that Rapa reduced the isoproterenol-induced increases in the thicknesses of left ventricular anterior and posterior walls during systole (LVAWs, LVPWs) (LVAWs: $2.52 \pm 0.21$ versus $3.22 \pm 0.20 ; P<0.001$ versus model; LVPWs: $2.80 \pm 0.27$ versus $3.22 \pm 0.16 ; P<0.05$ versus model). On the other hand, the cardiac function, which was represented by LVEF and LVFS, was not changed ( $P=$ ns versus model) (Figure $1(\mathrm{~d})$ ).
Additionally, real-time PCR analysis was performed to quantify the expression of hypertrophic marker genes including ANF, BNP, and $\beta$-MHC (Figure $1(\mathrm{e})$ ). As expected, levels of ANF, BNP, and $\beta$-MHC mRNA were increased about 2fold in model group $(P<0.05$ versus control). In contrast, Rapa treatment significantly decreased the expression of these marker genes, comparing with the model group $(P<$ 0.05 versus model).

3.2. Effects of Rapa on the Energy Homeostasis in Hypertrophied Hearts. TEM analyses were used to examine the ultrastructures of mitochondria. As seen from Figure 2(a), disorganized cristae and vacuoles of mitochondria were observed in the hypertrophied hearts; in contrast, the structure of mitochondria was well organized and the cristae stayed intact and evident in Rapa-treated hearts.

In the hypertrophied hearts treated with Rapa, state III respiration rates were significantly increased compared with those in hypertrophied hearts $(P<0.01$ versus model). This meant the dysfunction of mitochondria in the hypertrophied hearts could be attenuated by Rapa treatment (Figure 2(b)). Consistently, the production of ATP was significantly decreased in the hypertrophied hearts infused with isoproterenol, which was substantially reversed by Rapa treatment $(67.74 \pm 10.04$ versus $49.78 \pm 11.09, P<0.05$ versus model) (Figure $2(d)$ ), suggesting that the efficiency of energy supply was maintained by Rapa.

In order to investigate the role of Rapa in regulating fatty acid metabolism genes in hypertrophied hearts, we subsequently performed quantitative real time-PCR analysis among experimental groups. As shown in Figure 2(e), the treatment of Rapa prevented the declines in representative metabolic target genes associated with fatty acid metabolism, including carnitine palmitoyl transferase- $1 \beta$ and -2 (CPT$1 \beta$ and CPT-2), and medium- and long-chain acyl-CoA dehydrogenase (MCAD and LCAD) $(P<0.05$ versus model).

\subsection{The Activation of $N F-\kappa B$ Pathway in Hypertrophied} Hearts Was Inhibited by Rapa Treatment. Upon activation by hypertrophic stimuli, p65, the subunit of NF- $\kappa \mathrm{B}$, rapidly enters the nucleus, which resulted in elevated expression of hypertrophic genes [22]. We next examined the effects of Rapa treatment on the nuclear translocation of p65 and the degradation of $\mathrm{I} \kappa \mathrm{B}-\alpha$ in adult rats. The persistent stimulation of isoproterenol significantly promoted the translocation of p65 from cytoplasm to the nucleus, which were prevented by Rapa treatment $(P<0.05$ versus model) (Figures 2(f) and $2(\mathrm{~g}))$. Consistently, as shown in Figures $2(\mathrm{~h})$ and 2(i), the degradation of $\mathrm{I} \kappa \mathrm{B}-\alpha$ was detected to increase in response to isoproterenol stimulation ( $P<0.05$ versus control), and this effect can be reversed by Rapa $(P<0.05$ versus model).

3.4. Effects of Rapa on Proinflammatory Cytokines Release and Gene Expression in Cardiomyocytes via NF- $\kappa$ B Pathway. P65 was knocked down in neonatal rat cardiomyocytes using siRNA, and TNF- $\alpha$, a cytokine known to activate NF- $\kappa \mathrm{B}$ signal pathway, was used to further clarify the role of NF$\kappa \mathrm{B}$ in the protective effects of Rapa in cardiomyocytes. It has 

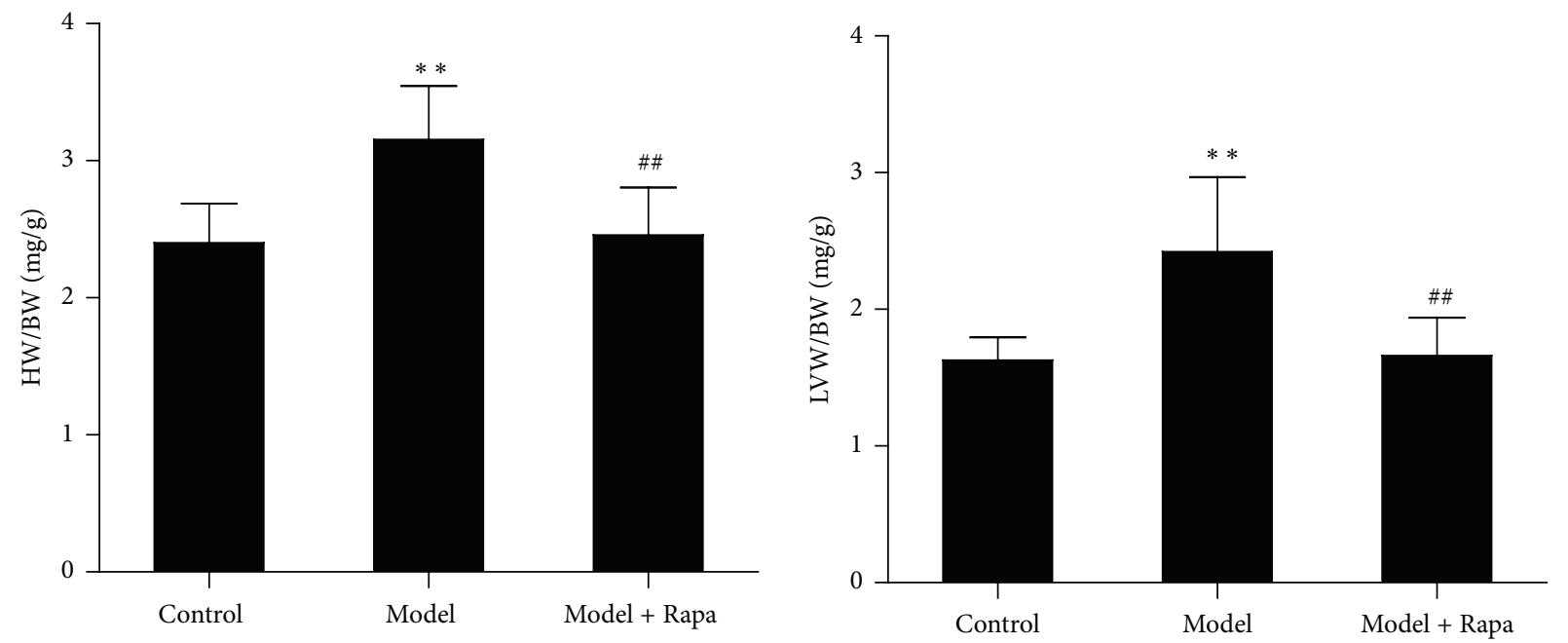

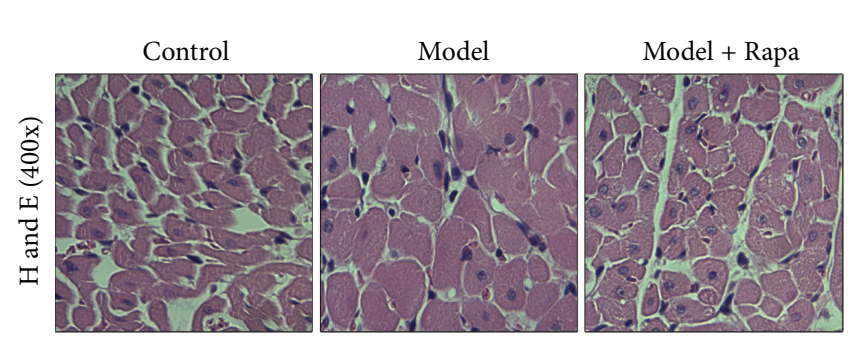

(b)

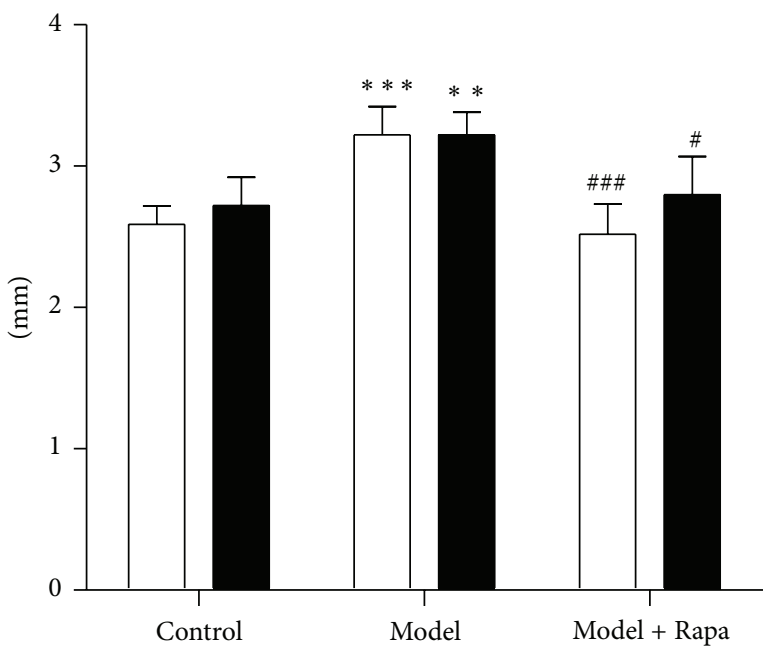

LVAWs

LVPWs (a)

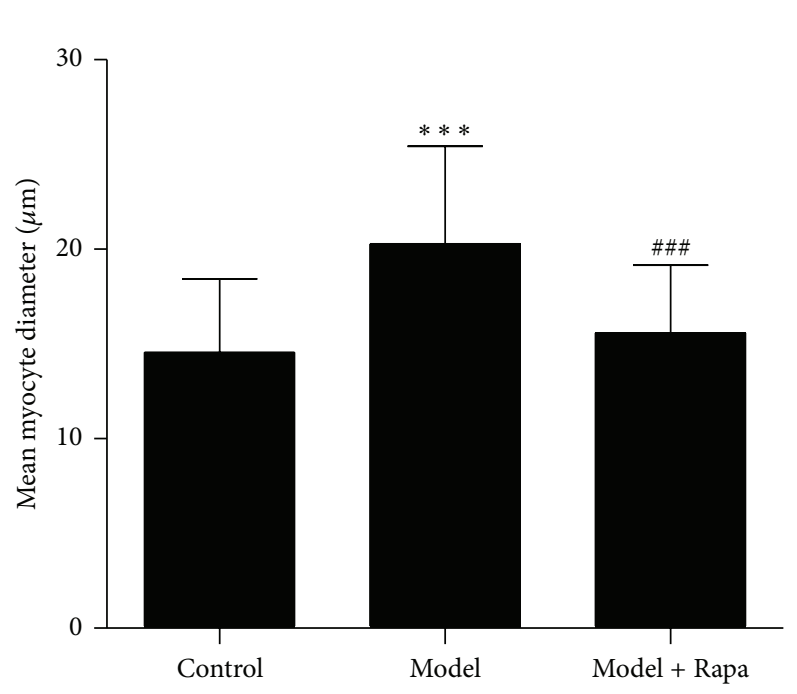

(c)

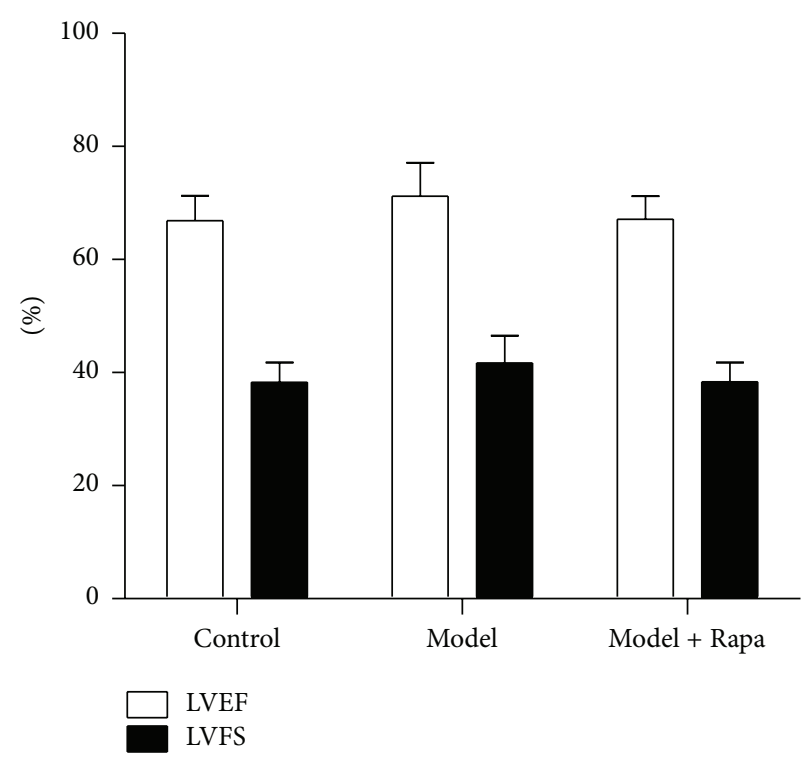

(d)

Figure 1: Continued. 


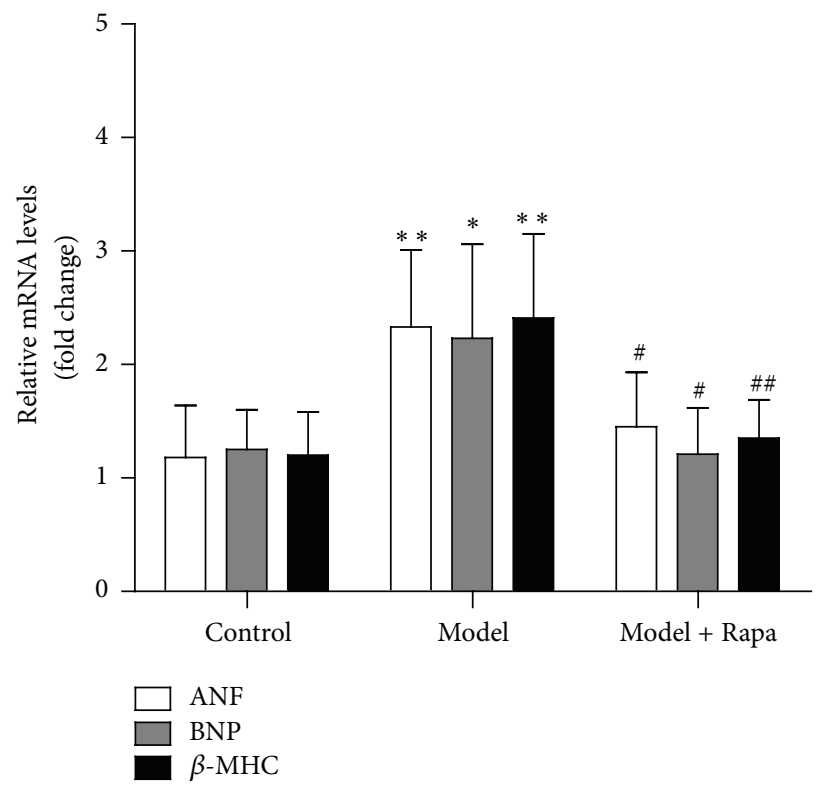

(e)

FIGURE 1: Rapa attenuated cardiac hypertrophy induced by consecutive infusion of isoproterenol. (a) Changes in the ratio of heart weight to body weight (HW/BW) and the ratio of left ventricular weight to body weight (LVW/BW). Values represented mean \pm SD of 6 rats in each group. (b) Representative high magnification $(\times 400)$ of left ventricular stained with hematoxylin and eosin from each group. $(c)$ Mean myocyte diameter was calculated by randomly measuring 100 cells from 5 sections in each group. (d) The thickness of left ventricular anterior and posterior wall (left) and left ventricular fraction shortening and left ventricular ejection fraction. Echocardiographic measurements were performed, and values represented as mean \pm SD of 6 rats in each group. (e) Gene expression patterns of ANF, BNP, and $\beta$-MHC among different groups. The mRNA was prepared and normalized to GAPDH gene. Model, the cardiac hypertrophy model induced by isoproterenol; Rapa, isoproterenol-infused rats treated with Rapa. Values represented as mean $\pm \mathrm{SD}$ of 4 to 5 rats in each group. ${ }^{*} P<0.05$ versus control group; ${ }^{* *} P<0.01$ versus control group; ${ }^{* * *} P<0.001$ versus control group; ${ }^{\#} P<0.05$ versus model group; ${ }^{\# \#} P<0.01$ versus model group; and ${ }^{\# \# \#} P<0.001$ versus model group.

been shown that the protein expression of p65 in cultured cardiomyocytes was increased by TNF- $\alpha$ in a concentrationdependent manner by western blot analysis (see Supplementary Figure 1(a) in Supplementary Material available online at http://dx.doi.org/10.1155/2014/868753), and p65 depletion was proved to decrease the protein expression of p65 (Supplementary Figure 1(c)) in cardiomyocytes.

To investigate whether the activation of the NF- $\kappa \mathrm{B}$ pathway is implicated in proinflammatory gene expressions and cytokines releases induced by isoproterenol, the extracellular production of cytokines was measured by ELISA. As shown in Figures 3(a), 3(b), and 3(c), releases of IL-1 $\beta$, IL2 , and TNF- $\alpha$ were significantly induced by isoproterenol $(P<0.01$ versus control). In contrast, the administration of Rapa to cardiomyocytes attenuated the increases in releases of proinflammatory cytokines $(P<0.05$ versus model), and this effect could be abrogated by TNF- $\alpha(20 \mathrm{ng} / \mathrm{mL})$. Then real-time PCR analysis was performed to quantify the expression of representative cytokine genes. The stimulation of isoproterenol led to increases in mRNA levels of IL$1 \beta$, IL-2, and TNF- $\alpha(P<0.05$ versus control), which could be reversed by Rapa (Figure 3(d)). Furthermore, it was revealed that this downregulation of proinflammatory cytokines by Rapa was reversed by TNF- $\alpha(20 \mathrm{ng} / \mathrm{mL})$, whereas the anti-inflammatory effect of Rapa was preserved when NF- $\kappa \mathrm{B} / \mathrm{p} 65$ was depleted in cardiomyocytes.

3.5. NF- $\kappa B$ Participates in the Antihypertrophic Effect of Rapa. In order to explore whether the activation of the NF- $\kappa$ B pathway is implicated in the underlying mechanism of the inhibitory effects of Rapa on cardiac hypertrophy, immunofluorescence analyses and real-time PCR analyses were performed in primary cultured neonatal cardiomyocytes. As shown in Figures 4(a) and 4(b), the surface area of cardiomyocytes, as well as mRNA levels of hypertrophied maker genes, was increased significantly in response to isoproterenol stimulation for 24 hours $(P<0.05$ versus control). In contrast, the administration of Rapa to cardiomyocytes attenuated the increases in hypertrophy biomarkers $(P<0.05$ versus model), and this protective effect could be abrogated by TNF- $\alpha(20 \mathrm{ng} / \mathrm{mL})$. In contrast, this antihypertrophic effect of Rapa was maintained after p65 depletion in cardiomyocytes. These results suggested the involvement of NF$\kappa \mathrm{B}$ inactivation in the protective effect of Rapa against cardiac hypertrophy induced by isoproterenol.

3.6. The Role of Rapa in Maintaining Energy Homeostasis Was Dependent on NF- $\kappa B$. To investigate the role of NF- $\kappa \mathrm{B}$ 


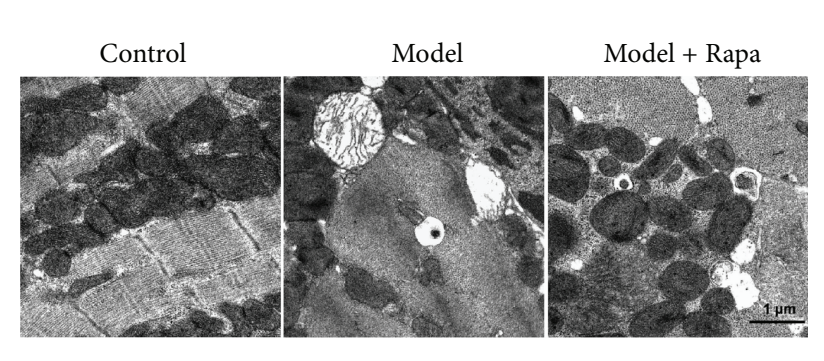

(a)

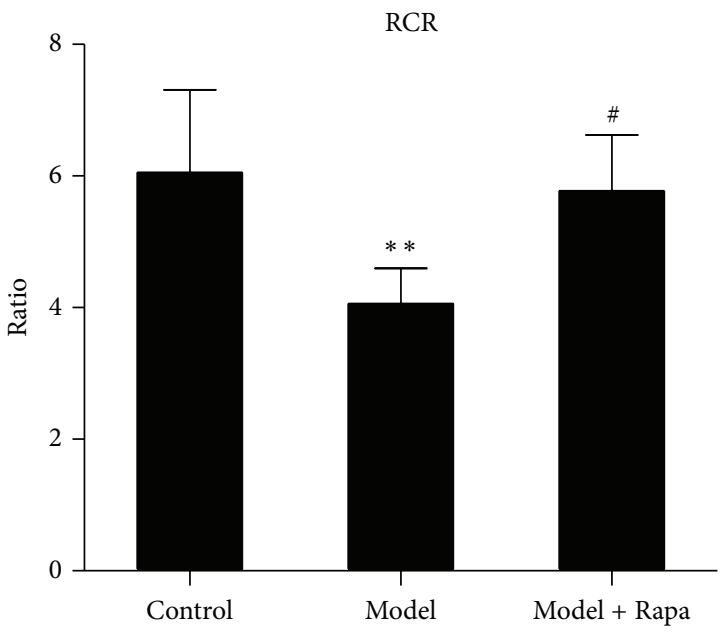

(c)

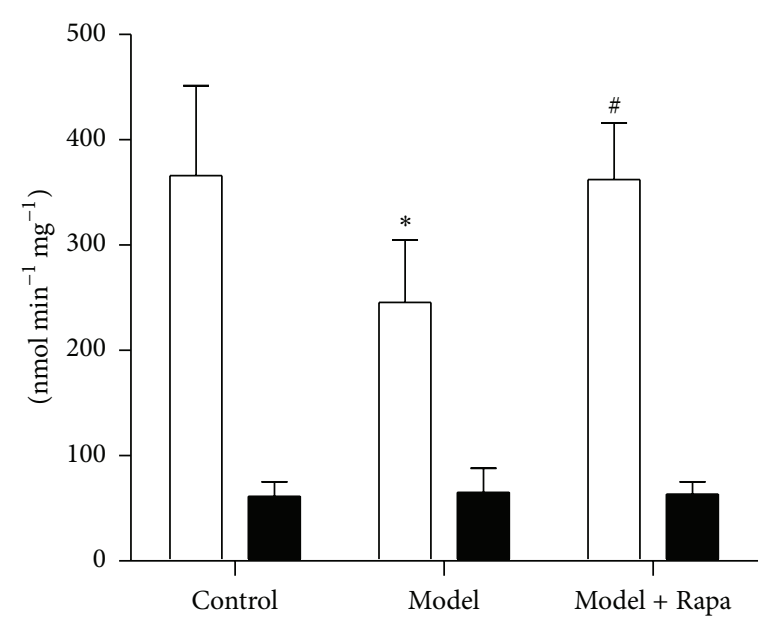

State 3 respiration rates State 4 respiration rates

(b)

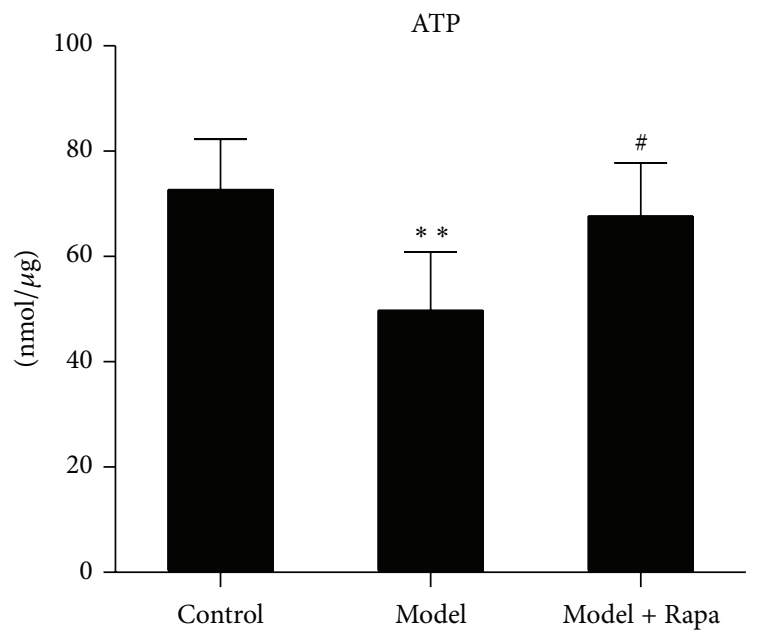

(d)

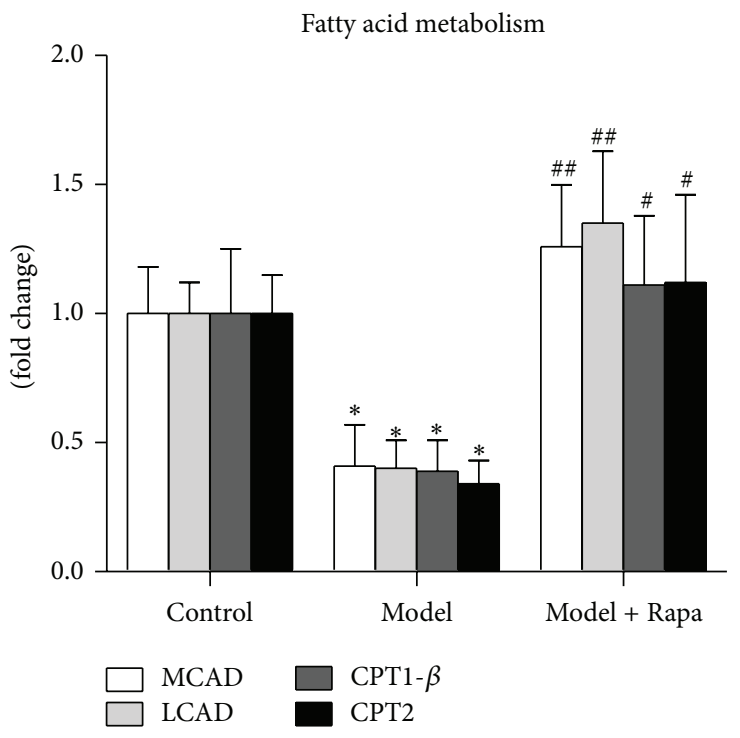

(e)

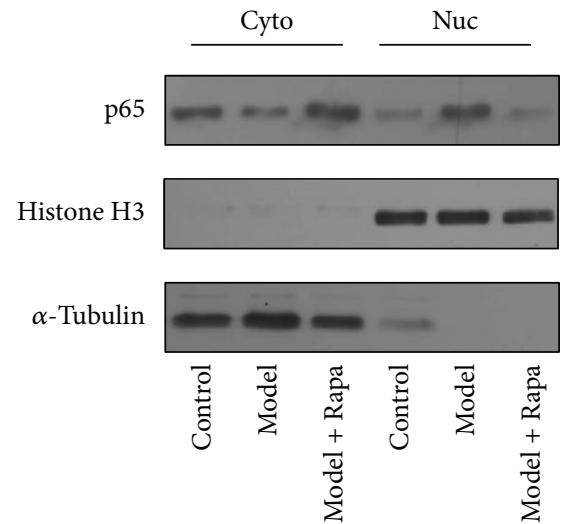

(f)

Figure 2: Continued. 


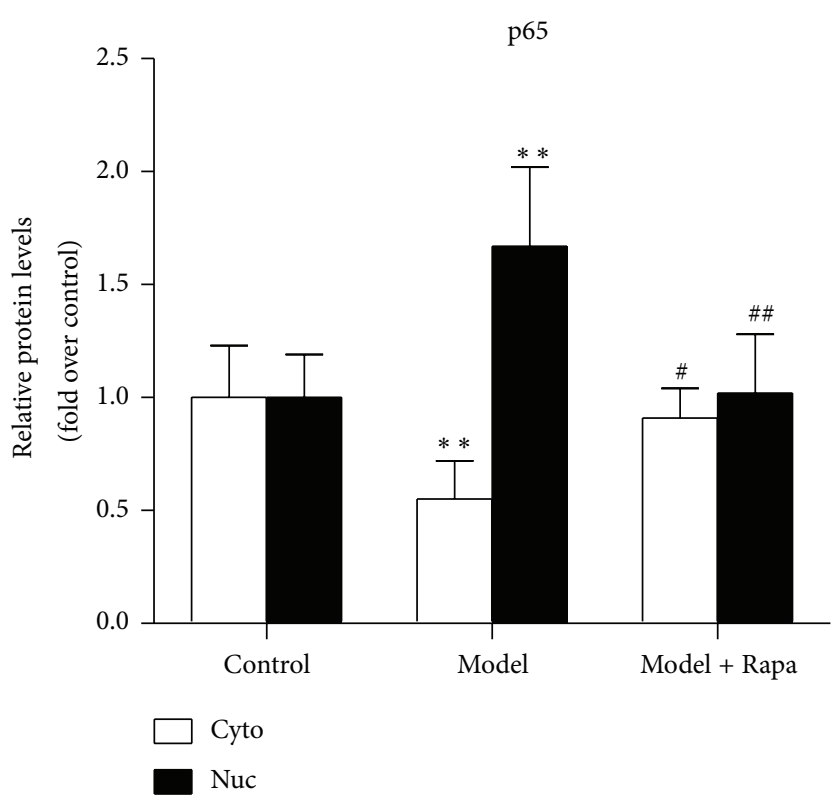

(g)

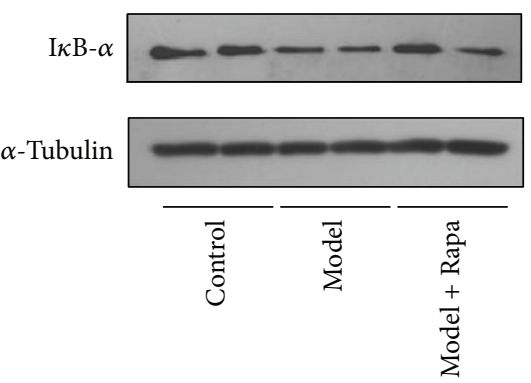

(h)

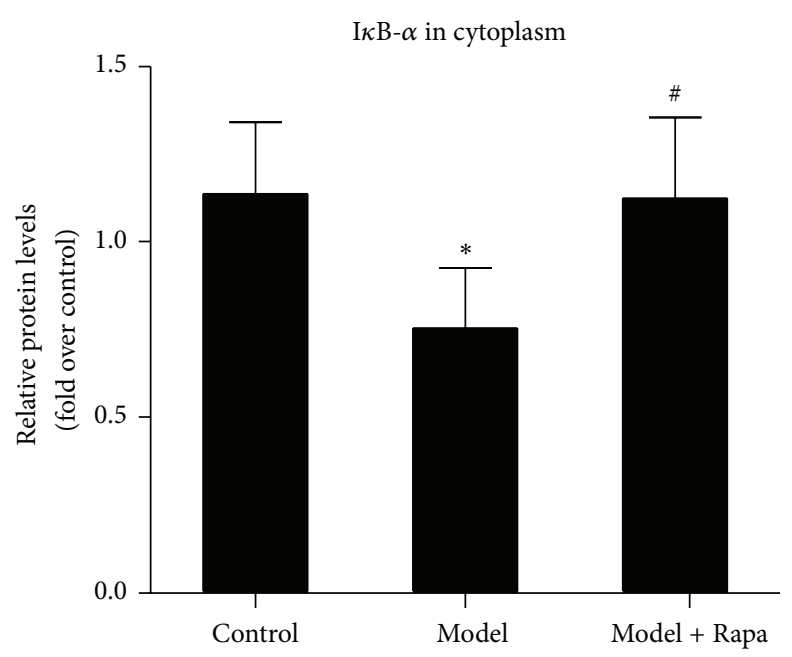

(i)

FIGURE 2: Rapa maintained energy homeostasis and inhibited NF- $\kappa$ B activation in hypertrophied hearts induced by isoproterenol. (a) The ultrastructures of the mitochondria were analyzed under TEM. Transmission electron micrographs of histological sections from the apex of left ventricle with different treatments were shown. Black arrows indicate disorganized cristae and vacuoles of mitochondria in the hypertrophied hearts. White arrows indicate autophagosomes. Scale bars, $1 \mu \mathrm{m}$. (b) State III and state IV respiration rates. (c) Respiratory control rate (RCR) was calculated as the ratio of state III respiration rates to state IV respiration rates. Values represented as mean \pm SD of 6 rats in each group. (d) ATP production. Values represented as mean \pm SD of 6 rats in each group. (e) Expression of metabolic genes associated with fatty acid metabolism at mRNA levels. The mRNA was prepared and normalized to $\beta$-actin gene. Values represented as mean \pm SD of 4 to 5 rats in each group. ((f) and (h)) Cytoplasmic and nuclear extracts from left ventricles of three groups of rats were immunoblotted with anti-p65 and anti-I $\kappa \mathrm{B}-\alpha$ antibodies. ((g) and (i)) Densitometric analysis of the relative protein expression of p65 and I $\kappa \mathrm{B}-\alpha$. Histone H3 and $\alpha$-tubulin were used to normalize the nuclear and cytosolic proteins loading, respectively. Values represented as mean \pm SD of 4 to 5 rats in each group. Model, the cardiac hypertrophy model induced by isoproterenol; Rapa, isoproterenol-infused rats treated with Rapa. ${ }^{*} P<0.05$ versus control group; ${ }^{* *} P<0.01$ versus control group; ${ }^{\#} P<0.05$ versus model group; and ${ }^{\# \#} P<0.01$ versus model group.

pathway in preserving mitochondrial function in cardiomyocytes, TMRE, a sensitive fluorescent probe that reflects the level of membrane potential, were used to assess the capacity of mitochondria to produce ATP. As seen from Figure 4(c), a significant loss of membrane potential was shown in cardiomyocytes treated with isoproterenol $(P<0.001$ versus control), which could be abolished partially by Rapa $(P<$ 0.01 versus model). Consistent with TMRE staining, the ATP production in cardiomyocytes was decreased notably in response to isoproterenol stimulation, compared with vehicle 


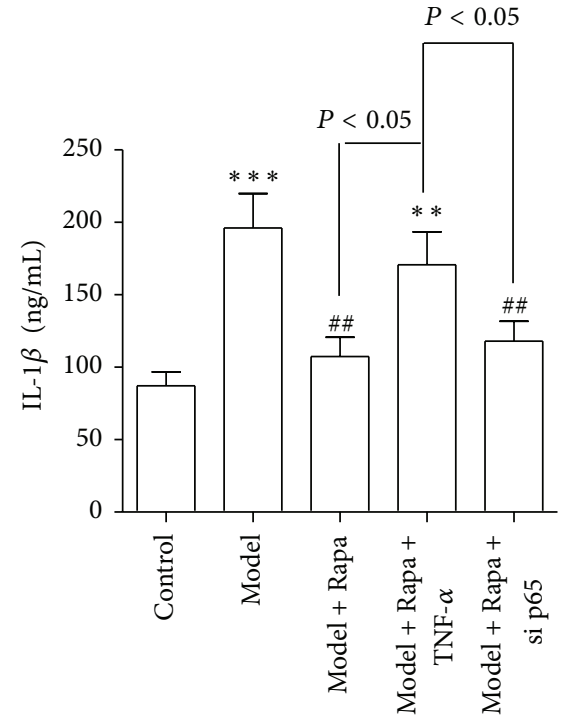

(a)

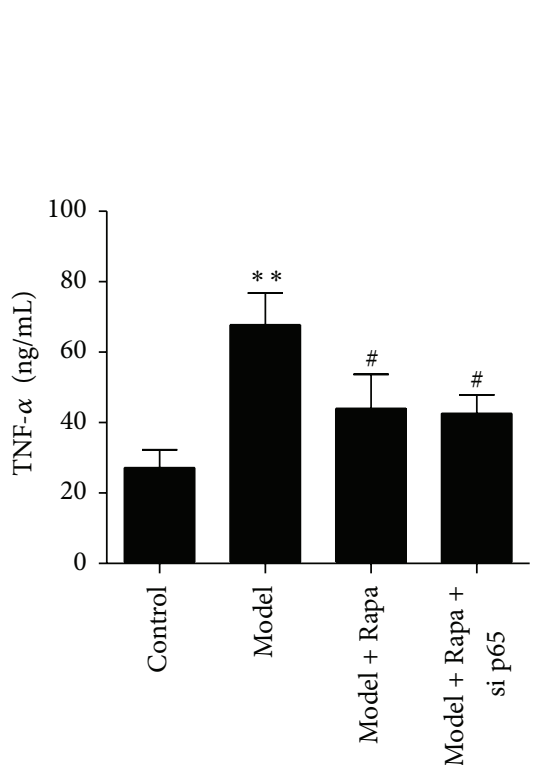

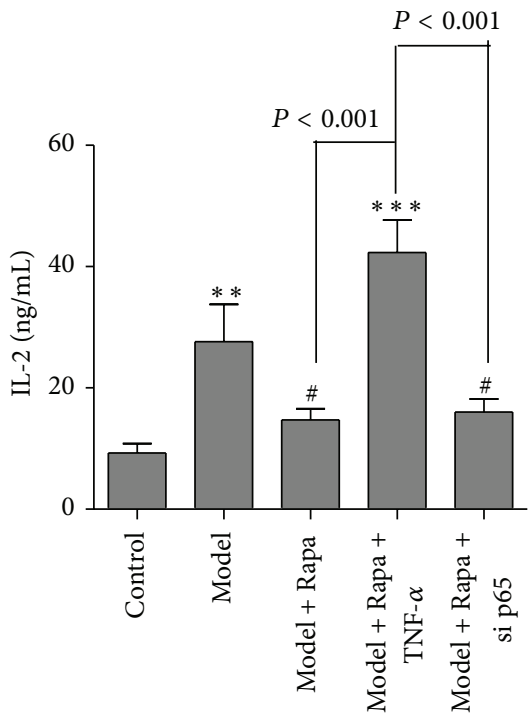

(b)

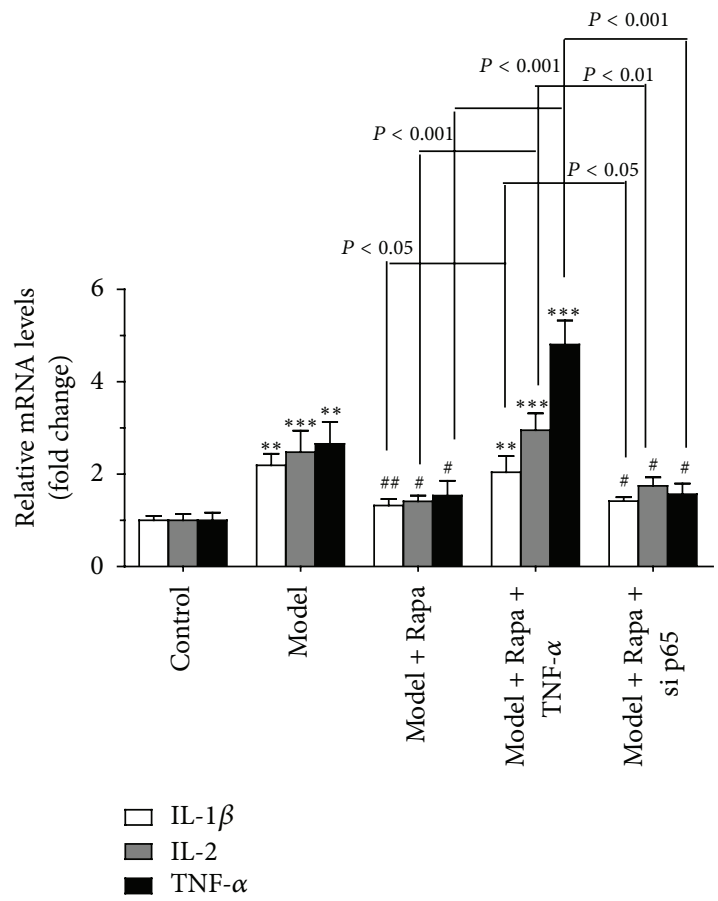

(d)

FIGURE 3: NF- $\kappa$ B participates in the role of Rapa in reducing pro-inflammatory cytokines release and gene expressions in cardiomyocytes ((a), (b), and (c)) ELISA was used to determine the release of IL-1 $\beta$, IL-2 and TNF- $\alpha$ into the cultured medium. (d) Real-time PCR analysis was performed to quantify the mRNA levels of proinflammatory cytokines. The mRNA was prepared and normalized to $\beta$-actin gene. $n=3$ experiments in duplicate. Error bars, SD. ${ }^{* *} P<0.01$ versus control group; ${ }^{* * *} P<0.001$ versus control group; ${ }^{\#} P<0.05$ versus model group; and ${ }^{\# \#} P<0.01$ versus model group.

control $(P<0.01$ versus control). And this reduction in conversion of metabolic substrates into usable energy can be substantially attenuated by Rapa $(P<0.05$ versus model) (Figure 4(d)). Moreover, it was observed that the effect of Rapa on preserving mitochondrial function and ATP production in hypertrophied cardiomyocytes was abrogated by TNF- $\alpha$ (20 ng/mL). In contrast, the protective role of Rapa in maintaining energy homeostasis was preserved after p65 depletion in cardiomyocytes.

3.7. The Role of Rapa in Upregulating Genes Associated with Fatty Acid Metabolism Was Dependent on NF- $\kappa B$. In order to explore the mechanisms underlying the protective role of Rapa in maintaining energy homeostasis, the activation of 

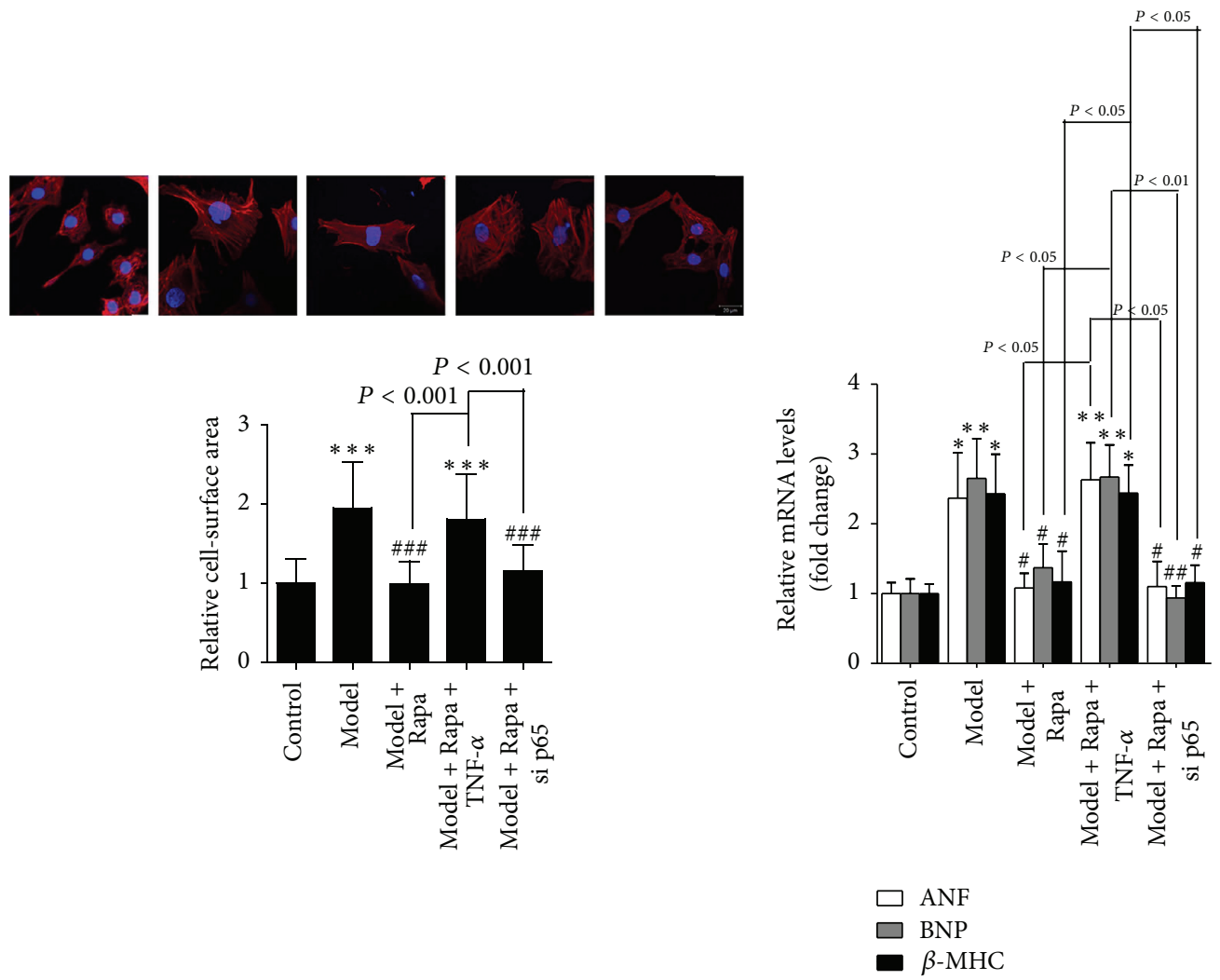

(a)

(b)
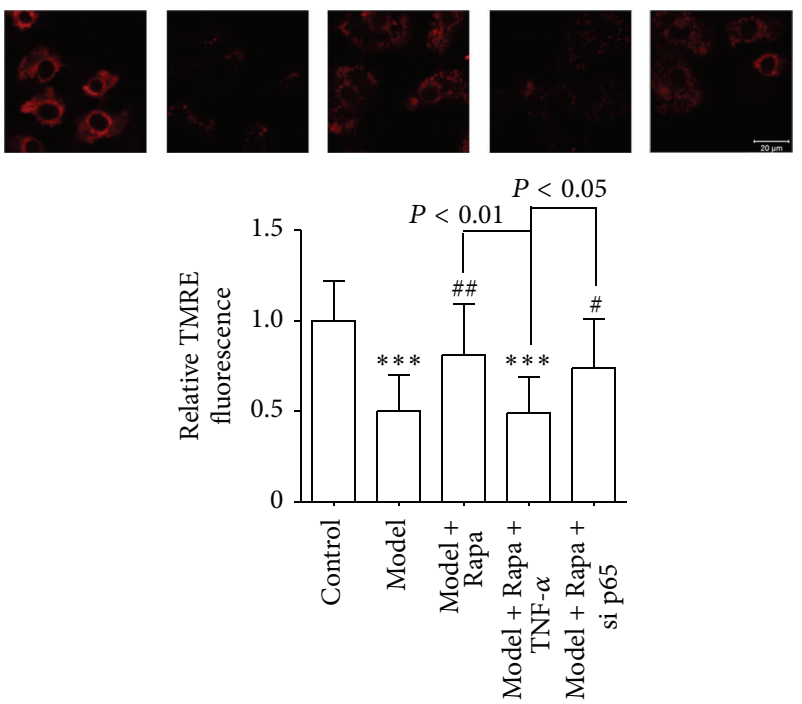

(c)

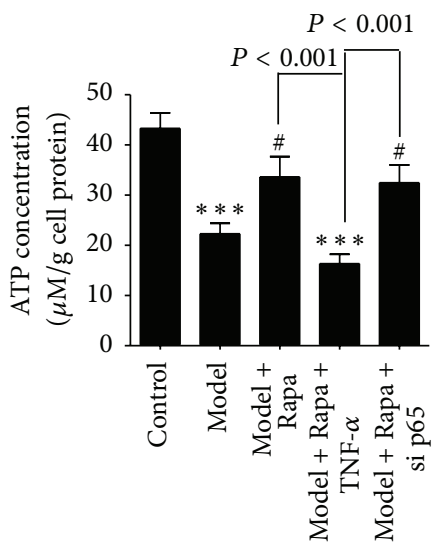

(d)

FIGURE 4: NF- $\kappa$ B participates in the protective role of Rapa in regressing cardiac hypertrophy and energy homeostasis. (a) Cardiac myocytes were stained with rhodamine-phalloidin followed by cell surface area quantitation. (b) Gene expression pattern of atrial natriuretic factor (ANF), brain natriuretic peptide (BNP), and $\beta$-myosin heavy chain ( $\beta$-MHC) among different groups. The mRNA was prepared and normalized to GAPDH gene. (c) Fluorescence of mitochondrial in TMRE loaded active cardiac myocytes under different treatments. The relative values of TMRE fluorescence intensity was normalized to 1.0. Scale bar, $20 \mu \mathrm{m}$. The results were obtained from three independent experiments. (d) ATP production. $n=3$ experiments in duplicate. Error bars, SD. ${ }^{*} P<0.05$ versus control group; ${ }^{* *} P<0.01$ versus control group; ${ }^{* * *} P<0.001$ versus control group; ${ }^{\#} P<0.05$ versus model group; ${ }^{\# \#} P<0.01$ versus model group; and ${ }^{\# \# \#} P<0.001$ versus model group. 
mTOR, which serves as a sensor of the energy state [23], was determined by western blot analysis. It was observed that the administration of isoproterenol to cardiomyocytes for 24 hours decreased the phosphorylation of mTOR at the site of serine 2448 ( $P<0.01$ versus control), which could be substantially attenuated by Rapa (Figures 5(a) and 5(b)). Furthermore, the mRNA and protein expression of its downstream transcriptional factor PPAR $\alpha$ and coactivator PGC- $1 \alpha$ were determined. As shown in Figures $5(\mathrm{c}), 5(\mathrm{~d})$, and 5(e), the downregulation of PPAR $\alpha$ and PGC- $1 \alpha$ in both mRNA and protein levels could be inhibited by Rapa $(P<$ 0.05 versus model), and these effects of Rapa were abrogated by $\mathrm{TNF}-\alpha(20 \mathrm{ng} / \mathrm{mL})$.

Besides, the mRNA levels of medium- and long-chain acyl-CoA dehydrogenase (MCAD and LCAD) and carnitine palmitoyl transferase- $1 \beta$ and -2 (CPT- $1 \beta$ and CPT-2) were notably decreased by isoproterenol, which could be partially reversed by Rapa $(P<0.01$ versus model); however, the effect of Rapa on upregulating these genes associated with fatty acid metabolism was abrogated by the pretreatment of TNF- $\alpha$ $(20 \mathrm{ng} / \mathrm{mL}$ ) (Figures 5(f) and 5(g)). Collectively, these results suggested the involvement of $\mathrm{NF}-\kappa \mathrm{B}$ inactivation in the role of Rapa in upregulating genes associated with fatty acid metabolism in cardiomyocytes treated with isoproterenol.

\section{Discussion}

In this study, it was demonstrated that the regression of cardiac hypertrophy by Rapa in adult rat was associated with attenuation of the increases in myocyte cell size and HW/BW, without loss of left ventricular function. Activated NF- $\kappa \mathrm{B}$ pathway was detected in the hypertrophied hearts, which was substantially reversed by Rapa. Moreover, Rapa protected cardiomyocytes from the mitochondrial dysfunction and abnormal energy utilization, which could be attributed to the inactivation of NF- $\kappa \mathrm{B}$. Conversely, activation of NF- $\kappa \mathrm{B}$ pathway resulted in the catastrophic loss of ATP production in cultured cardiomyocytes and abrogated the protective role of Rapa in regressing cardiac hypertrophy. Our results revealed the mechanisms underlying the protective role of Rapa against cardiac hypertrophy induced by isoproterenol, suggesting that blocking proinflammatory response by Rapa might contribute to the maintenance of energy homeostasis during the progression of cardiac hypertrophy.

Long-lived postmitotic cells, including neurons and cardiomyocytes, are most vulnerable to mitochondrial dysfunction [24]. In order to evaluate the effect of Rapa on the functional performance of mitochondria in hypertrophied hearts, a well-established method was performed [25]. RCR, which reflects the mitochondrial function, decreased significantly in the hypertrophied hearts induced by isoproterenol (Figure 2(c)), and these functional changes were associated with evidences of structural abnormalities in mitochondria (Figure 2(a)). And consistently, these injuries on structure and functional performance of mitochondria caused by persistent stimulation of isoproterenol could be substantially attenuated by Rapa.
NF- $\kappa \mathrm{B}$ had been demonstrated to be involved in pathogeneses of inflammatory disorders [26] and viewed as a link between cardiomyopathy and the dysregulation of energy metabolism [27]. In this study, we observed that the administration of Rapa to the hypertrophied hearts could reverse the activation of NF- $\kappa$ B induced by isoproterenol (Figure 2(f)). Rapa was demonstrated to inhibit mTOR activity through a mechanism involving interaction with FKBP12 [28]. And prior to our study, this inhibition of mTOR was proved to suppress the activity of I- $\kappa \mathrm{B}$ kinase (an essential activator of NF$\kappa \mathrm{B})$ through a mechanism that might involve dissociation of Raptor from mTOR [29]. In keeping with these observations, we found that the degradation of $\mathrm{I} \kappa \mathrm{B}-\alpha$ induced by isoproterenol was substantially attenuated by Rapa administration (Figure $2(\mathrm{~h})$ ), thereby maintaining NF- $\kappa \mathrm{B}$ in an inactive state. Herein, we found that the administration of isoproterenol to cardiomyocytes led to an aberrant production of varieties of proinflammatory cytokines (Figures 3(a), 3(b), and 3(c)), which was in line with the activation of NF- $\kappa \mathrm{B}$ pathway induced by isoproterenol. The nuclear translocated NF- $\kappa \mathrm{B}$ was demonstrated to regulate the expression of various genes which encode proinflammatory cytokines including TNF- $\alpha$ [30]. Meanwhile, the downregulation of cytokine releases by Rapa was impaired under TNF- $\alpha$ treatment, indicating that the role of Rapa in restraining the inflammatory responses in cardiomyocytes under persistent stimulation of isoproterenol was NF- $\kappa \mathrm{B}$-dependent.

In the present study, the stimulation of isoproterenol promoted inflammatory responses in hypertrophied cardiomyocytes and Rapa could partially restrain the downregulation of PPAR $\alpha$ induced by isoproterenol (Figure 5(a)). One potential explanation to this effect of Rapa in upregulating $\operatorname{PPAR} \alpha$ may be its activity in inactivation of mTOR, just as we found in the decrease in the ratio of phosphormTOR to total mTOR (Figure 5(b)). Recently, it has been reported that pharmacological or genetic inhibition of mTORC1 was able to promote the translocation of transcription factor EB (TFEB) to the nucleus and increase its transcription activity [31]. More importantly, it was demonstrated that TFEB controls cellular lipid metabolism trough a mechanism of upregulation of PGC- $1 \alpha$ and PPAR $\alpha$ [32], which were proved to play prominent roles in controlling genes mediating process from fatty acid uptake to oxidation, including medium-chain acyl-CoA dehydrogenase (MCAD) and carnitine palmitoyl transferase (CPT) $[33,34]$.

Herein, the present results revealed that the administration of isoproterenol to hearts weakened the expression of CPT- $1 \beta$ and CPT-2, which were proved to be crucial in mediating the rate-limiting reaction of fatty acid transport [35], and this effect could be partially abrogated by Rapa (Figure 2(e)). Moreover, clusters of genes associated with fatty acid metabolism downregulated by isoproterenol in hypertrophied cardiomyocytes were activated by Rapa, which could be abrogated by TNF- $\alpha$. In contrast, the role of Rapa in upregulating these genes was maintained by inactivation of NF- $\kappa \mathrm{B}$ in cardiomyocytes (Figures $5(\mathrm{f})$ and $5(\mathrm{~g})$ ). Our observations reconciled with those findings concerning that TNF- $\alpha$ impaired fatty acid oxidation in the heart through 


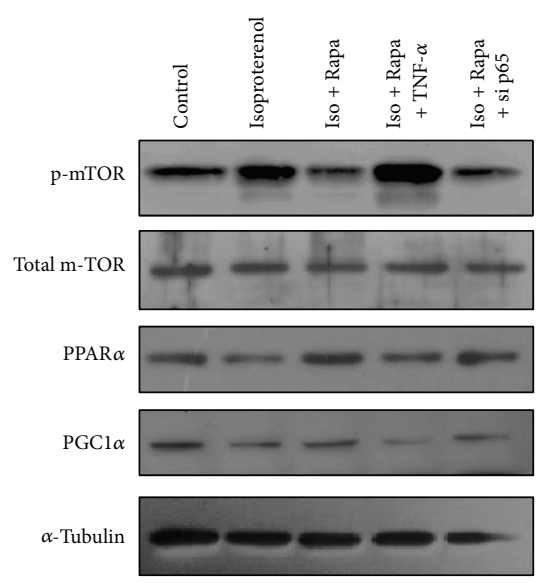

(a)

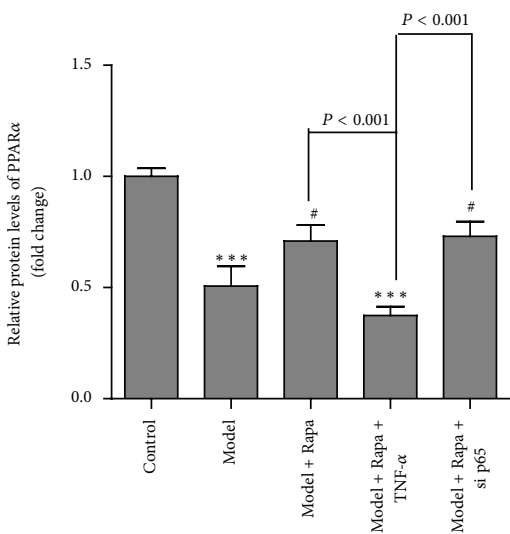

(c)

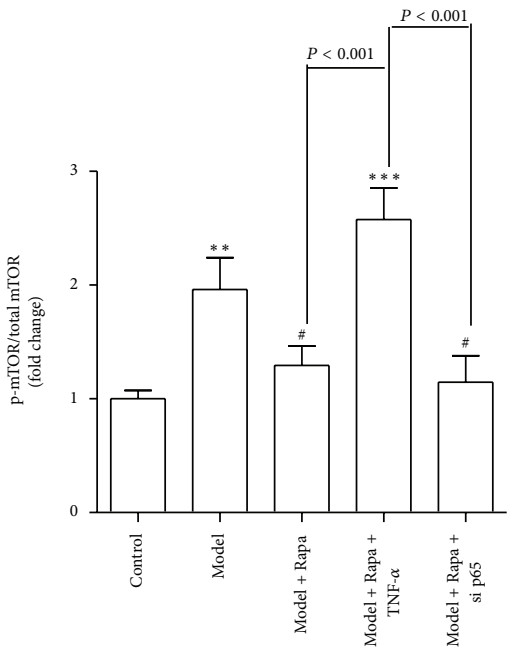

(b)

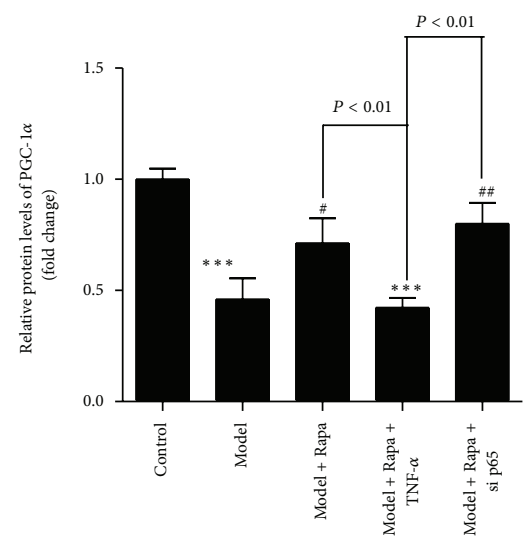

(d)

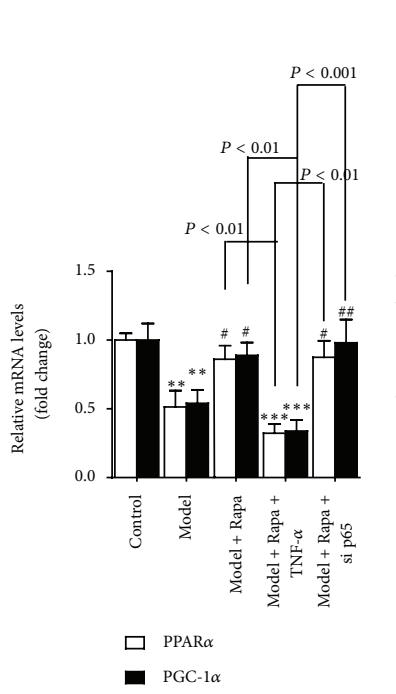

(e)

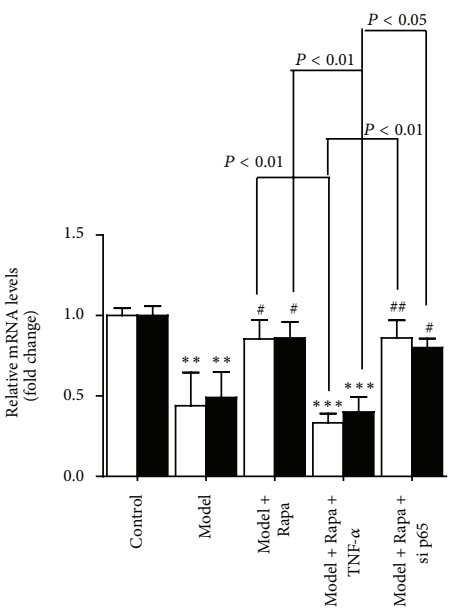

$\square$ MCAD

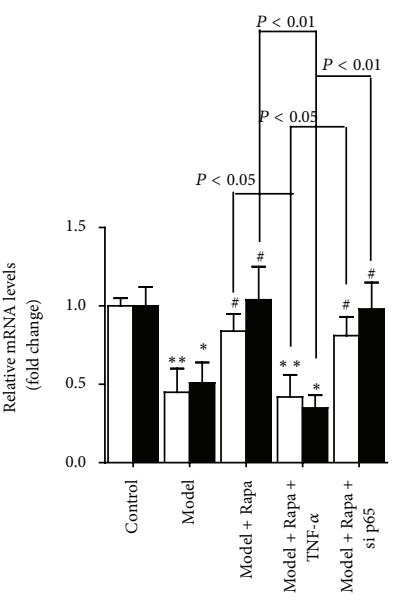

$\square$ CPT1 $\beta$

- $\mathrm{CPT2}$

(g)

FIGURE 5: NF- $\kappa$ B participates in the role of Rapa in upregulating fatty acid metabolism in cardiomyocytes induced by isoproterenol. (a) Cytoplasmic proteins extracted from cardiomyocytes were immunoblotted with anti-p-mTOR, mTOR, PPAR $\alpha$, and PGC-1 $\alpha$ antibodies. ((b), (c), and (d)) Densitometric analysis of the relative protein expression of the ratio of p-mTOR/mTOR, PPAR $\alpha$, and PGC-1 $\alpha$. And $\alpha$-tubulin was used to normalize proteins loading. ((d), (e), and (f)) Expression of metabolic genes associated with fatty acid metabolism (PPAR $\alpha$, PGC1 $\alpha$, MCAD, LCAD, CPT- $1 \beta$, and CPT- 2 ) at mRNA levels. The mRNA was prepared and normalized to $\beta$-actin gene. $n=3$ experiments in duplicate. Error bars, SD. ${ }^{*} P<0.05$ versus control group; ${ }^{* *} P<0.01$ versus control group; ${ }^{*} P<0.001$ versus control group; ${ }^{*} P<0.05$ versus model group; and ${ }^{\#} P<0.01$ versus model group. 
activation of NF- $\kappa$ B, which underlies cardiac dysfunction and heart failure in metabolic diseases [36].

It has been reported that the activation of S6 Kinase was critical for the development of cardiac hypertrophy in response to pressure overload. And Rapa was able to attenuate this induction of cardiac hypertrophy, which could be attributed to the inhibition of S6 Kinase [37, 38]. Similarly, previous in vitro studies had reported that Rapa was able to attenuate angiotensin-II-induced cardiac hypertrophy through inactivation of S6 Kinase [39]. These results indicated that the role of Rapa in regressing of cardiac hypertrophy induced by pressure-overload or angiotensin-II was associated with inactivation of S6 Kinase, which plays an important role in increases in overall protein synthesis in cardiomyocytes. In the present study, we established the model of cardiac hypertrophy induced by consecutive infusion of isoproterenol to mimic the sympathetic stimulation [40]. Herein, proinflammatory response was associated with energy metabolism alterations in the model of cardiac hypertrophy induced by isoproterenol. Rapa was demonstrated to ameliorate energy homeostasis in cardiomyocytes via inactivation of NF- $\kappa \mathrm{B}$, thereby attenuating established cardiac hypertrophy after isoproterenol stimulation. These findings suggested that blocking proinflammatory response by Rapa might contribute to the maintenance of energy homeostasis and provided rational basis for development of novel strategies to prevent cardiac hypertrophy after sympathetic stimulation.

In this study, although the thickness of LVAW increased markedly in the rat model of cardiac hypertrophy induced by isoproterenol, the left ventricular function (represented by LVEF and LVFS) was maintained. One potential explanation to this paradox might be the compensatory ability of the heart, and it would develop heart failure after the infusion of isoproterenol for longer time [41]. And our findings were consistent with the reported data that there was no significant decrease in cardiac function of the hypertrophic heart [42]. Herein, we observed that during the compensated state of cardiac hypertrophy induced by isoproterenol, Rapa was able to attenuate cardiac remodeling and maintained energy homeostasis without loss of cardiac function in adult rat. This present study reconciled with the earlier findings that autophagy activated by Rapa reduced endotoxininduced inflammatory responses in epithelial cells, maintaining intestinal homeostasis ultimately [43]. Interestingly, it has been reported that the administration of Rapa was less effective in regressing decompensated cardiac hypertrophy in mice than compensated cardiac hypertrophy ( $40 \%$ and $70 \%$ regression, resp.) [38]. Therefore, further studies are required to investigate mechanisms underlying the difference of Rapa in treating cardiac hypertrophy under different states.

In summary, these present in vivo and in vitro studies demonstrated the protective role of Rapa in isoproterenolinduced cardiac hypertrophy, which was dependent on NF$\kappa \mathrm{B}$ pathway. These findings may provide clues to the pathophysiology of proinflammatory response associated with energy metabolism alterations during the progression of cardiac hypertrophy and shed new light on the underlying mechanism of Rapa in protecting hearts from cardiac hypertrophy under the persistent stimulation of neurohormonal factors.

\section{Abbreviations}

$\begin{array}{ll}\text { Rapa: } & \text { Rapamycin } \\ \text { mTOR: } & \text { Mammalian target of rapamycin } \\ \text { HW: } & \text { Heart weight } \\ \text { LVW: } & \text { Left ventricle weight } \\ \text { BW: } & \text { Body weight } \\ \text { LVEF: } & \text { Left ventricular ejection fraction } \\ \text { LVFS: } & \text { Left ventricular fractional shortening } \\ \text { LVAW: } & \text { Left ventricular anterior wall } \\ \text { LVPW: } & \text { Left ventricular posterior wall } \\ \text { RCR: } & \text { Respiratory control rate } \\ \text { ANF: } & \text { Atrial natriuretic factor } \\ \text { BNP: } & \text { Brain natriuretic peptide } \\ \text { MHC: } & \text { Myosin heavy chain } \\ \text { NF- } \kappa \text { B: } & \text { Nuclear factor-kappa B } \\ \text { TEM: } & \text { Transmission electron microscopy } \\ \text { FBS: } & \text { Fetal calf serum } \\ \text { DMEM: } & \text { Dulbecco Modified Eagle Medium } \\ \text { TRITC: } & \text { Tetraethyl rhodamine isothiocyanate } \\ \text { TNF- } \alpha: & \text { Tumor necrosis factor- } \alpha \\ \text { TMRE: } & \text { Tetramethylrhodamine ethyl ester } \\ \text { PPAR: } & \text { Peroxisome proliferator-activated receptor } \\ \text { PGC-1 } \alpha: & \text { PPAR } \gamma \text { coactivator-1 } \alpha .\end{array}$

\section{Conflict of Interests}

The authors have no conflict of interests to disclose.

\section{Authors' Contribution}

Xi Chen and Siyu Zeng contributed equally to this paper.

\section{Acknowledgments}

This work was supported by grants from the National Natural Science Foundation of China (nos. 81072641; 81273499). The authors gratefully acknowledge Kunpeng Li (Electron Microscopy Research Laboratory, Sun Yat-sen University) for excellent assistance with the TEM analysis.

\section{References}

[1] B. H. Lorell and B. A. Carabello, "Left ventricular hypertrophy: pathogenesis, detection, and prognosis," Circulation, vol. 102, no. 4, pp. 470-479, 2000.

[2] P. M. Okin, D. A. Hille, S. E. Kjeldsen, B. Dahlof, and R. B. Devereux, "Persistence of left ventricular hypertrophy is associated with increased cardiovascular morbidity and mortality in hypertensive patients with lower achieved systolic pressure during antihypertensive treatment," Blood Pressure, vol. 23, no. 2, pp. 71-80, 2013.

[3] S. Neubauer, "The failing heart-an engine out of fuel," The New England Journal of Medicine, vol. 356, no. 11, pp. 1140-1151, 2007. 
[4] G. D. Lopaschuk, J. R. Ussher, C. D. L. Folmes, J. S. Jaswal, and W. C. Stanley, "Myocardial fatty acid metabolism in health and disease," Physiological Reviews, vol. 90, no. 1, pp. 207-258, 2010.

[5] A. Terman and U. T. Brunk, "Myocyte aging and mitochondrial turnover," Experimental Gerontology, vol. 39, no. 5, pp. 701-705, 2004.

[6] M. G. Rosca, B. Tandler, and C. L. Hoppel, "Mitochondria in cardiac hypertrophy and heart failure," Journal of Molecular and Cellular Cardiology, vol. 55, no. 1, pp. 31-41, 2013.

[7] I. T. Meredith, G. Eisenhofer, G. W. Lambert, E. M. Dewar, G. L. Jennings, and M. D. Esler, "Cardiac sympathetic nervous activity in congestive heart failure: evidence for increased neuronal norepinephrine release and preserved neuronal uptake," Circulation, vol. 88, no. 1, pp. 136-145, 1993.

[8] J. D. Bisognano, H. D. Weinberger, T. J. Bohlmeyer et al., "Myocardial-directed overexpression of the human $\beta 1$ adrenergic receptor in transgenic mice," Journal of Molecular and Cellular Cardiology, vol. 32, no. 5, pp. 817-830, 2000.

[9] K. Rivard, P. Paradis, M. Nemer, and C. Fiset, "Cardiac-specific overexpression of the human type 1 angiotensin II receptor causes delayed repolarization," Cardiovascular Research, vol. 78, no. 1, pp. 53-62, 2008.

[10] M. Shimizu, H. Sasaki, J. Sanjo et al., "Effect of captopril on isoproterenol-induced cardiac hypertrophy and polyamine contents," Japanese Circulation Journal, vol. 56, no. 11, pp. 11301137, 1992.

[11] R. N. Saunders, M. S. Metcalfe, and M. L. Nicholson, "Rapamycin in transplantation: a review of the evidence," Kidney International, vol. 59, no. 1, pp. 3-16, 2001.

[12] H. Kanamori, G. Takemura, K. Goto et al., "The role of autophagy emerging in postinfarction cardiac remodelling," Cardiovascular Research, vol. 91, no. 2, pp. 330-339, 2011.

[13] E. Paoletti, M. Amidone, P. Cassottana, M. Gherzi, L. Marsano, and G. Cannella, "Effect of sirolimus on left ventricular hypertrophy in kidney transplant recipients: a 1-year nonrandomized controlled trial," American Journal of Kidney Diseases, vol. 52, no. 2, pp. 324-330, 2008.

[14] G. Y. Oudit, M. A. Crackower, U. Eriksson et al., "Phosphoinositide 3-kinase $\gamma$-deficient mice are protected from isoproterenolinduced heart failure," Circulation, vol. 108, no. 17, pp. 2147-2152, 2003.

[15] J. Zou, K. Le, S. Xu et al., "Fenofibrate ameliorates cardiac hypertrophy by activation of peroxisome proliferator-activated receptor- $\alpha$ partly via preventing $\mathrm{p} 65-\mathrm{NF} \kappa \mathrm{B}$ binding to NFATc4," Molecular and Cellular Endocrinology, vol. 370, no. 1-2, pp. 103112, 2013.

[16] W. Oriyanhan, H. Tsuneyoshi, T. Nishina, S. Matsuoka, T. Ikeda, and M. Komeda, "Determination of optimal duration of mechanical unloading for failing hearts to achieve bridge to recovery in a rat heterotopic heart transplantation model," Journal of Heart and Lung Transplantation, vol. 26, no. 1, pp. 1623, 2007.

[17] R. Singh, S. Kaushik, Y. Wang et al., "Autophagy regulates lipid metabolism," Nature, vol. 458, no. 7242, pp. 1131-1135, 2009.

[18] H. Bugger, M. Schwarzer, D. Chen et al., "Proteomic remodelling of mitochondrial oxidative pathways in pressure overload-induced heart failure," Cardiovascular Research, vol. 85, no. 2, pp. 376-384, 2010.

[19] J. J. Fu, H. Gao, R. B. Pi, and P. Q. Liu, "An optimized protocol for culture of cardiomyocyte from neonatal rat," Cytotechnology, vol. 49, no. 2-3, pp. 109-116, 2005.
[20] Y. Li, H. Zhang, W. Liao et al., "Transactivated EGFR mediates $\alpha$ 1-AR-induced STAT3 activation and cardiac hypertrophy," American Journal of Physiology: Heart and Circulatory Physiology, vol. 301, no. 5, pp. H1941-H1951, 2011.

[21] Y. Nakagawa, K. Kuwahara, G. Takemura et al., "P300 plays a critical role in maintaining cardiac mitochondrial function and cell survival in postnatal hearts," Circulation Research, vol. 105, no. 8, pp. 746-754, 2009.

[22] J. D. Molkentin, J.-R. Lu, C. L. Antos et al., "A calcineurindependent transcriptional pathway for cardiac hypertrophy," Cell, vol. 93, no. 2, pp. 215-228, 1998.

[23] T. P. Neufeld, "Contribution of Atgl-dependent autophagy to TOR-mediated cell growth and survival," Autophagy, vol. 3, no. 5, pp. 477-479, 2007.

[24] A. Terman, T. Kurz, M. Navratil, E. A. Arriaga, and U. T. Brunk, "Mitochondrial Turnover and aging of long-lived postmitotic cells: the mitochondrial-lysosomal axis theory of aging," Antioxidants and Redox Signaling, vol. 12, no. 4, pp. 503-535, 2010.

[25] A. Fritah, J. H. Steel, D. Nichol et al., "Elevated expression of the metabolic regulator receptor-interacting protein 140 results in cardiac hypertrophy and impaired cardiac function," Cardiovascular Research, vol. 86, no. 3, pp. 443-451, 2010.

[26] S. Vallabhapurapu and M. Karin, "Regulation and function of NF- $\kappa$ B transcription factors in the immune system," Annual Review of Immunology, vol. 27, pp. 693-733, 2009.

[27] A. Planavila, J. C. Laguna, and M. Vázquez-Carrera, "Nuclear factor- $\kappa \mathrm{B}$ activation leads to down-regulation of fatty acid oxidation during cardiac hypertrophy," The Journal of Biological Chemistry, vol. 280, no. 17, pp. 17464-17471, 2005.

[28] C. L. Sawyers, "Will mTOR inhibitors make it as cancer drugs?" Cancer Cell, vol. 4, no. 5, pp. 343-348, 2003.

[29] H. C. Dan, M. J. Cooper, P. C. Cogswell, J. A. Duncan, J. P.-Y. Ting, and A. S. Baldwin, "Akt-dependent regulation of NF- $\kappa$ B is controlled by mTOR and Raptor in association with IKK," Genes and Development, vol. 22, no. 11, pp. 1490-1500, 2008.

[30] K. J. Tracey, S. F. Lowry, and A. Cerami, “The pathophysiologic role of cachectin/TNF in septic shock and cachexia," Annales de l'Institut Pasteur Immunology, vol. 139, no. 3, pp. 311-317, 1988.

[31] J. A. Martina, Y. Chen, M. Gucek, and R. Puertollano, "MTORC1 functions as a transcriptional regulator of autophagy by preventing nuclear transport of TFEB," Autophagy, vol. 8, no. 6, pp. 903-914, 2012.

[32] C. Settembre, R. de Cegli, G. Mansueto et al., "TFEB controls cellular lipid metabolism through a starvation-induced autoregulatory loop," Nature Cell Biology, vol. 15, no. 8, pp. 647-658, 2013.

[33] B. N. Finck and D. P. Kelly, "Peroxisome proliferator-activated receptor $\gamma$ coactivator-1 (PGC-1) regulatory cascade in cardiac physiology and disease," Circulation, vol. 115, no. 19, pp. 25402548, 2007.

[34] K. Watanabe, H. Fujii, T. Takahashi et al., "Constitutive regulation of cardiac fatty acid metabolism through peroxisome proliferator-activated receptor $\alpha$ associated with age-dependent cardiac toxicity," The Journal of Biological Chemistry, vol. 275, no. 29, pp. 22293-22299, 2000

[35] M. A. Martín, M. A. Gómez, F. Guillén et al., "Myocardial carnitine and carnitine palmitoyltransferase deficiencies in patients with severe heart failure," Biochimica et Biophysica Acta, vol. 1502, no. 3, pp. 330-336, 2000.

[36] X. Palomer, D. Álvarez-Guardia, R. Rodríguez-Calvo et al., "TNF- $\alpha$ reduces PGC- $1 \alpha$ expression through NF- $\kappa$ B and p38 
MAPK leading to increased glucose oxidation in a human cardiac cell model," Cardiovascular Research, vol. 81, no. 4, pp. 703-712, 2009.

[37] T. Shioi, J. R. McMullen, O. Tarnavski et al., "Rapamycin attenuates load-induced cardiac hypertrophy in mice," Circulation, vol. 107, no. 12, pp. 1664-1670, 2003.

[38] J. R. McMullen, M. C. Sherwood, O. Tarnavski et al., "Inhibition of mTOR signaling with rapamycin regresses established cardiac hypertrophy induced by pressure overload," Circulation, vol. 109, no. 24, pp. 3050-3055, 2004.

[39] J. Sadoshima and S. Izumo, "Rapamycin selectively inhibits angiotensin II-induced increase in protein synthesis in cardiac myocytes in vitro: potential role of $70-\mathrm{kD}$ S6 kinase in angiotensin II-induced cardiac hypertrophy," Circulation Research, vol. 77, no. 6, pp. 1040-1052, 1995.

[40] F. Jaffré, J. Callebert, A. Sarre et al., "Involvement of the serotonin 5-HT2B receptor in cardiac hypertrophy linked to sympathetic stimulation: control of interleukin-6, interleukin$1 \beta$, and tumor necrosis factor- $\alpha$ cytokine production by ventricular fibroblasts," Circulation, vol. 110, no. 8, pp. 969-974, 2004.

[41] S. J. Matkovich, A. Diwan, J. L. Klanke et al., "Cardiac-specific ablation of G-protein receptor kinase 2 redefines its roles in heart development and $\beta$-adrenergic signaling," Circulation Research, vol. 99, no. 9, pp. 996-1003, 2006.

[42] O. Drews, O. Tsukamoto, D. Liem, J. Streicher, Y. Wang, and P. Ping, "Differential regulation of proteasome function in isoproterenol-induced cardiac hypertrophy," Circulation Research, vol. 107, no. 9, pp. 1094-1101, 2010.

[43] Y. Fujishima, S. Nishiumi, A. Masuda et al., "Autophagy in the intestinal epithelium reduces endotoxin-induced inflammatory responses by inhibiting NF- $\kappa \mathrm{B}$ activation," Archives of Biochemistry and Biophysics, vol. 506, no. 2, pp. 223-235, 2011. 


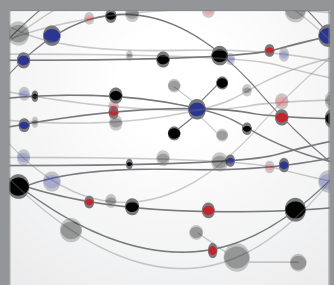

The Scientific World Journal
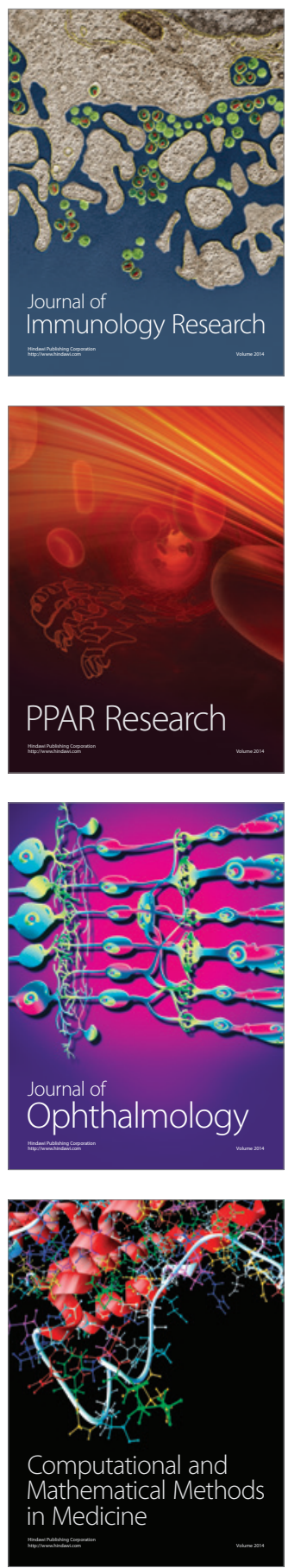

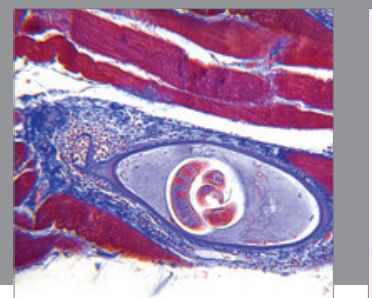

Gastroenterology

Research and Practice
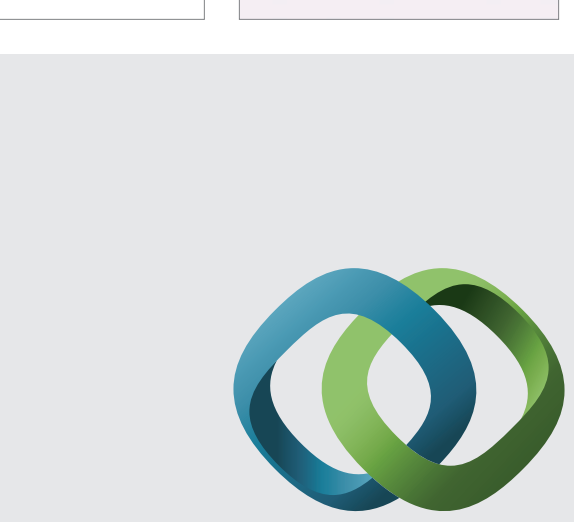

\section{Hindawi}

Submit your manuscripts at

http://www.hindawi.com
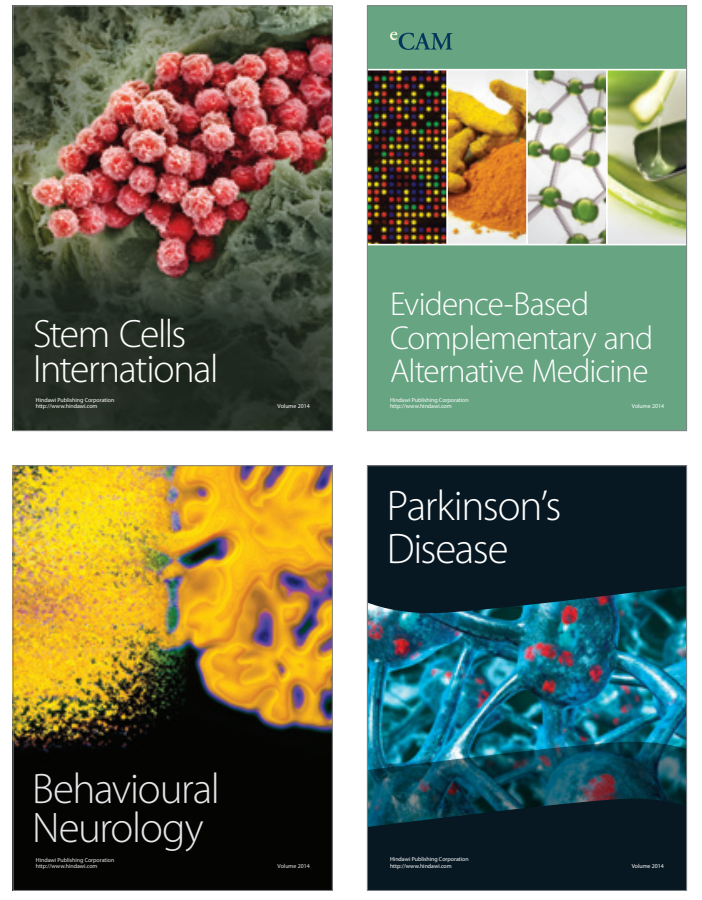
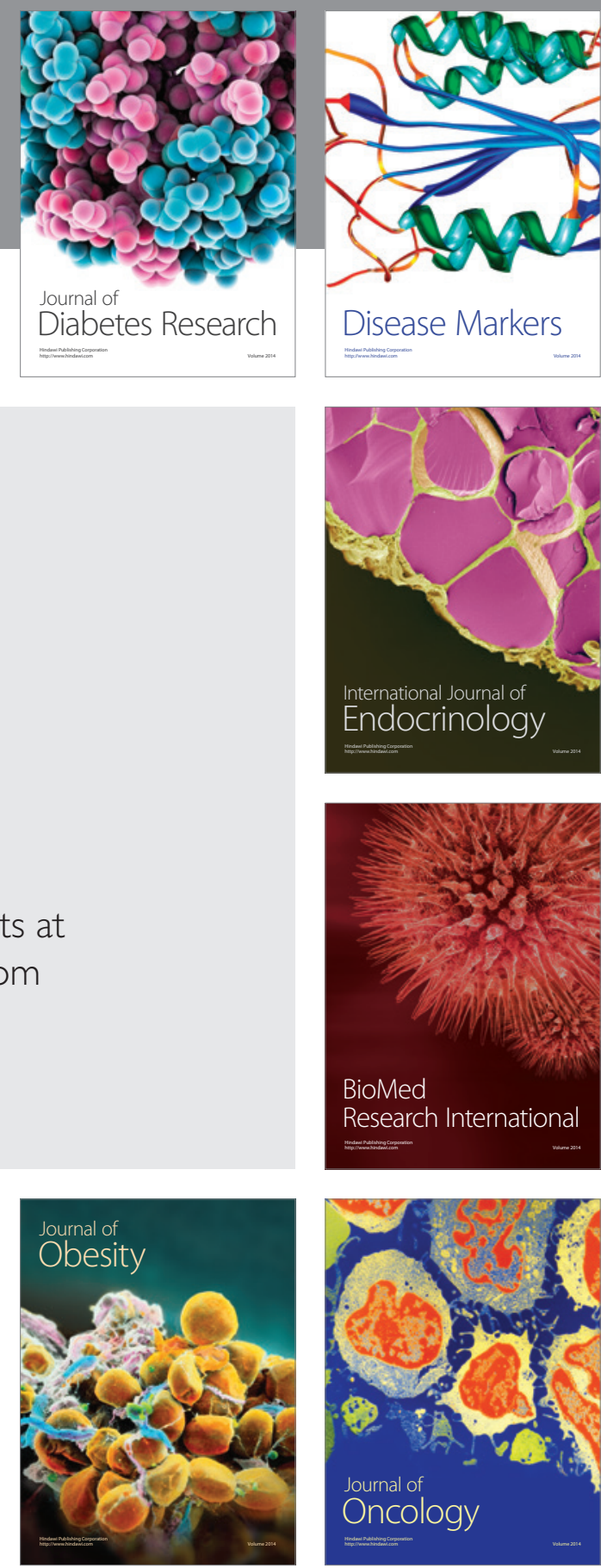

Disease Markers
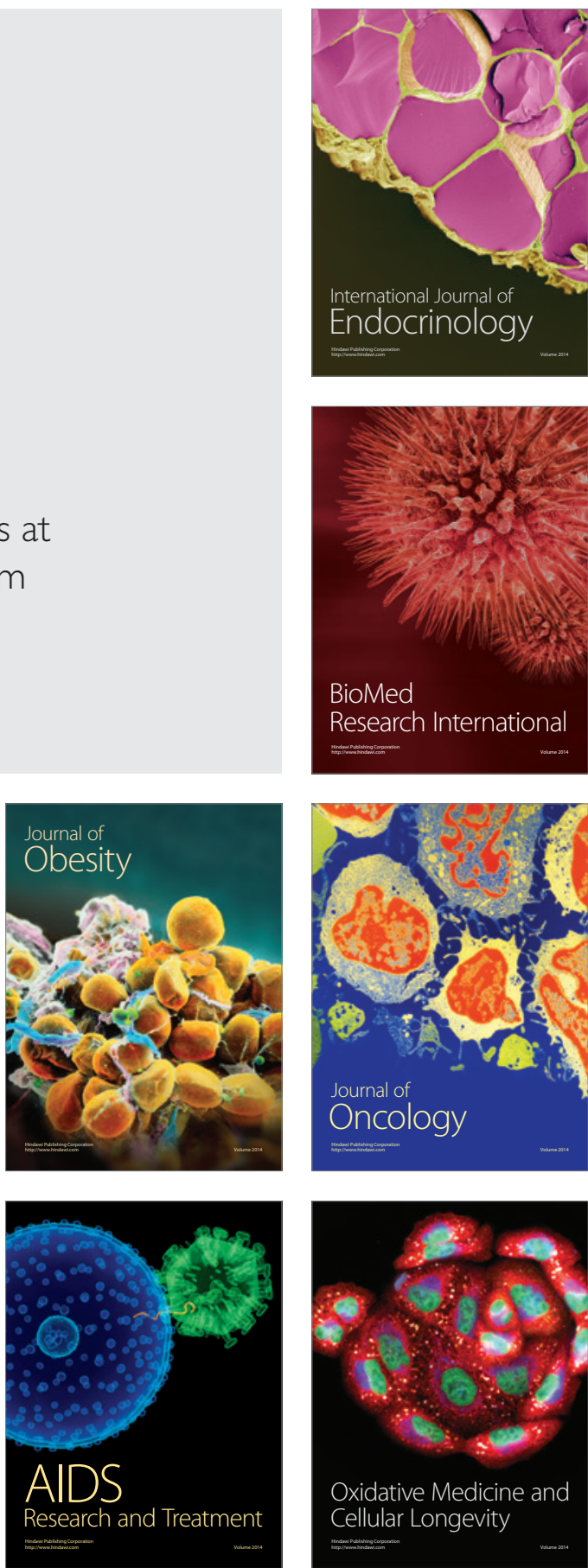